\title{
The decay of turbulence in rotating flows
}

\author{
Tomas Teitelbaum ${ }^{1}$ and Pablo D. Mininni ${ }^{1,2}$ \\ ${ }^{1}$ Departamento de Física, Facultad de Ciencias Exactas y Naturales, Universidad de Buenos Aires, \\ IFIBA and CONICET, Ciudad Universitaria, 1428 Buenos Aires, Argentina \\ ${ }^{2}$ National Center for Atmospheric Research, P.O. Box 3000, Boulder, Colorado 80307-3000, USA
}

(Received 8 September 2010; accepted 12 April 2011; published online 10 June 2011)

\begin{abstract}
We present a parametric space study of the decay of turbulence in rotating flows combining direct numerical simulations, large eddy simulations, and phenomenological theory. Several cases are considered: (1) the effect of varying the characteristic scale of the initial conditions when compared with the size of the box, to mimic "bounded" and "unbounded" flows; (2) the effect of helicity (correlation between the velocity and vorticity); (3) the effect of Rossby and Reynolds numbers; and (4) the effect of anisotropy in the initial conditions. Initial conditions include the Taylor-Green vortex, the Arn'old-Beltrami-Childress flow, and random flows with large-scale energy spectrum proportional to $k^{4}$. The decay laws obtained in the simulations for the energy, helicity, and enstrophy in each case can be explained with phenomenological arguments that consider separate decays for two-dimensional and three-dimensional modes and that take into account the role of helicity and rotation in slowing down the energy decay. The time evolution of the energy spectrum and development of anisotropies in the simulations are also discussed. Finally, the effect of rotation and helicity in the skewness and kurtosis of the flow is considered. (c) 2011 American Institute of Physics. [doi:10.1063/1.3592325]
\end{abstract}

\section{INTRODUCTION}

Nature presents several examples of rotating flows. Rotation influences large-scale motions in the Earth's atmosphere and oceans, as well as convective regions of the Sun and stars. Rotation is also important in many industrial flows, such as turbo machinery, rotor-craft, and rotating channels. In a rotating system, the Coriolis force, linear in the velocity, modifies the flow nonlinear dynamics when strong enough. In its presence, the Navier-Stokes equation becomes a multiscale problem with a "slow" time scale $\tau_{L} \sim L / U$ associated with the eddies at a characteristic scale $L$ ( $U$ is a characteristic velocity) and a "fast" time scale $\tau_{\Omega} \sim 1 / \Omega \sim \tau_{L} R o$ associated with inertial waves. The dimensionless Rossby number $R o$ is the ratio of advection to Coriolis forces and measures the influence of rotation upon the nonlinear dynamics of the system (decreasing as rotation becomes dominant).

Resonant wave theory provides a framework to study the effect of rapid rotation in turbulence. ${ }^{1-5}$ The separation between the fast and slow time scales results in a selection of the resonant triadic interactions as the ones responsible for the energy transfer among scales. As a result, energy transfer and dissipation are substantially decreased in the presence of strong rotation. ${ }^{3}$ The resonant condition is also responsible for the transfer of energy towards two-dimensional (2D) slow modes, driving the flow to a quasi-2D $\operatorname{state}^{3,4}$ (this result is often referred to in the literature as the "dynamic Taylor-Proudman theorem," see, e.g., Ref. 6). The development of anisotropy and reduction of the energy transfer and dissipation rates has been verified in numerical simulations $^{7-11}$ and experiments. ${ }^{12,13}$

Similar arguments (see, e.g., Ref. 14) indicate that in the limit of fast rotation (small Rossby number), the slow 2D modes decouple from the remaining fast three-dimen- sional (3D) modes and evolve under their own autonomous dynamics. Moreover, in that limit, the averaged equation for the slow modes splits into a 2D Navier-Stokes equation for the vertically averaged horizontal velocity and a passive scalar equation for the vertically averaged vertical velocity. Although simulations of forced rotating flows ${ }^{6}$ and of ideal truncated rotating flows ${ }^{15,16}$ using periodic boundary conditions show good agreement with these predictions for small enough Rossby numbers, for long times the decoupling of slow and fast modes seems to break down. Also, some authors ${ }^{17}$ argue that in unbounded domains no decoupling is achievable even for $R o=0$.

Of particular importance for many geophysical and astrophysical problems is how turbulence decays in time. The problem is also important for laboratory experiments, as it provides, e.g., one way to measure changes in the energy dissipation rate associated with the presence of rotation. Even in the absence of rotation, the decay of isotropic turbulence proves difficult to tackle because of the different decay laws obtained depending on boundary and initial conditions. As an example, for bounded flows (i.e., flows for which the initial characteristic length is close to the size of the vessel) the energy decays as $\sim t^{-2}$ (see, e.g., Refs. 13, 18, and 19). For unbounded flows (i.e., flows in an infinite domain, or in practice, flows for which the initial characteristic length is much smaller than the size of the vessel), a $\sim t^{-10 / 7}$ (Refs. 20 and 21) or $\sim t^{-6 / 5}$ (Refs. 13, 22, and 23) decay law is observed depending on whether the initial energy spectrum at large scales behaves as $\sim k^{4}$ or $\sim k^{2}$, respectively.

In the presence of rotation, the decay of turbulence becomes substantially richer, with decay rates depending not only on whether turbulence is bounded or unbounded and on the initial spectrum at large scales but also, e.g., on the strength of background rotation (see Ref. 24). A detailed 
experimental study of this dependence can be found in Refs. 12 and 13, where the authors studied the energy decay of grid-generated turbulence in a rotating tank using particle image velocimetry and found different decay laws depending upon the rate of rotation and the saturation (or not) of the characteristic size of the largest eddies. For large Rossby number, they reported a decay $\sim t^{\alpha}$ with exponent $\alpha \approx-1.1$ for non-rotating turbulence (consistent with the value of $-6 / 5$ predicted in Refs. 22 and 23 for $\sim k^{2}$ unbounded turbulence), which later turned to $\alpha \approx-2$ after the largest eddies grew to the experiment size. For small Rossby number, this energy decay rate became smaller saturating at $\approx-1$. Similar results were reported in simulations in Ref. 10, where a decrease from $\approx-10 / 7$ to $-5 / 7$ was observed as rotation was increased. These results are consistent with the reduction of the energy transfer discussed above. They also reported a steeper energy spectrum together with positive vorticity skewness for the rotating flows and anisotropic growth of integral scales (see also Refs. 7 and 25).

The decay rate of rotating turbulence also seems to depend on the helicity content of the flow. Helicity (the alignment between velocity and vorticity) is an ideal invariant of the equations of motion (a quantity conserved in the inviscid limit) with intriguing properties. In the absence of rotation, the presence of helicity does not modify the energy decay rate ${ }^{19,26,27}$ nor the dissipation rate. ${ }^{28}$ However, in the presence of rotation Ref. 27 reported a further decrease of the energy transfer when both rotation and helicity were present and Ref. 19 showed that the decay rate of bounded rotating flows changes drastically depending on whether helicity is present or not.

In this paper, we conduct a detailed study of parameter space of rotating helical flows, taking into account (1) the effect of varying the characteristic scale of the initial conditions when compared with the size of the box, (2) the effect of helicity, (3) the effect of Rossby and Reynolds numbers, and (4) the effect of anisotropy in the initial conditions. The numerical study uses a two pronged approach combining direct numerical simulations (DNS) and large eddy simulations (LES). Several initial conditions are considered, although when the characteristic initial scale of the flow is smaller than the size of the domain, we focus only on the case with large scale initial energy spectrum $\sim k^{4}$. The different decay laws obtained (which in some cases coincide with previous experimental or numerical observations, while in others are new) are explained using phenomenological arguments, and we classify the results depending on the relevant effects on each case.

After studying the decay laws, we study the evolution of anisotropy, skewness, and kurtosis, and the formation of columnar structures in the flow. We consider how helicity affects the evolution of skewness and kurtosis and associate peaks observed in the time evolution of these quantities with the dynamics of the columnar structures.

The structure of the paper is as follows. In Sec. II, we introduce the equations, describe DNS and LES, and give details of the initial conditions and different parameters used. Section III presents phenomenological arguments to obtain decay laws in turbulent flows with and without rota- tion. The phenomenological predictions are then compared with the numerical results in Sec. IV, which presents the energy, helicity, and enstrophy decay in all runs. The spectral evolution and development of anisotropy in the flows is discussed at the end of that section. The effect of initial anisotropy in the decay is considered in Sec. V. A statistical analysis including evolution of skewness and kurtosis is presented in Sec. VI. Finally, Sec. VII gives our conclusions.

\section{EQUATIONS AND MODELS}

\section{A. Equations}

The evolution of an incompressible fluid in a rotating frame is described by the Navier-Stokes equation with the Coriolis force

$$
\partial_{t} \mathbf{u}+\omega \times \mathbf{u}+2 \Omega \times \mathbf{u}=-\nabla \mathcal{P}+\nu \nabla^{2} \mathbf{u}
$$

and the incompressibility condition

$$
\nabla \cdot \mathbf{u}=0
$$

where $\mathbf{u}$ is the velocity field, $\omega=\nabla \times \mathbf{u}$ is the vorticity, the centrifugal term is included in the total pressure per unit of mass $\mathcal{P}$, and $\nu$ is the kinematic viscosity (uniform density is assumed). The rotation axis is in the $z$ direction, so $\boldsymbol{\Omega}=\boldsymbol{\Omega} \hat{z}$, where $\Omega$ is the rotation frequency.

As mentioned in the Introduction, these equations are solved numerically using two different methods: DNS and LES using a dynamical subgrid-scale spectral model of rotating turbulence that also takes into account the helicity cascade. All simulations were performed in a three dimensional periodic box of length $2 \pi$, using different spatial resolutions ranging from $96^{3}$ grid points for the lowest resolution LES runs up to $512^{3}$ for the highest resolution DNS.

\section{B. Models}

In a DNS, all spatial and time scales (up to the dissipation scale) are explicitly resolved. The simulations were performed using a parallelized pseudo-spectral code ${ }^{29,30}$ with the two-third rule for dealiasing. As a result, the maximum wave number resolved in the DNS is $k_{\max }=N / 3$, where $N$ is the linear resolution; to properly resolve the dissipative scales, the condition $k_{\eta} / k_{\max }<1$ must be satisfied during all simulations, where $k_{\eta}$ is the dissipation wave number. In practice, this condition is more stringent if reliable data about velocity gradients and high-order statistics of the flow are needed (see, e.g., Ref. 31, where they indicate $k_{\eta} / k_{\max }<0.5$ for such studies).

The dissipation wave number as a function of time was computed for all simulations in two different ways: as the Kolmogorov dissipation wave number for isotropic and homogeneous turbulence $k_{\eta}=\left(\epsilon / \nu^{3}\right)^{1 / 4}=\left(\left\langle\omega^{2}\right\rangle / \nu^{2}\right)^{1 / 4}$ (where $\epsilon$ is the energy dissipation rate and $\left\langle\omega^{2}\right\rangle$ is the mean square vorticity) and as the wave number where the enstrophy spectrum peaks. The Kolmogorov dissipation wave number was found to be always larger than the wave number where dissipation peaks, and in the following, we therefore only consider 
the Kolmogorov scale as it gives a more stringent condition on the resolution.

In all DNS discussed below, the ratio $k_{\eta} / k_{\max }$ was $\leq 0.7$ at the time of maximum dissipation $(t \approx 1$ to $t \approx 3$ depending on the simulation), 0.2 to 0.5 at $t \approx 10$ (when the self-similar decay starts in most runs), and monotonously decreases to values between 0.05 and 0.2 at $t \approx 100$. The spatial resolutions used were $256^{3}$ and $512^{3}$ grid points, and an explicit second-order Runge-Kutta method was used to evolve in time, with a Courant-Friedrichs-Levy (CFL) number smaller than one.

In the LES approach, only the large scales are explicitly resolved, while the statistical impact on the resolved scales of scales smaller than a cut-off scale is modeled with simplified equations. To this end, we use the spectral model derived in Ref. 32 for isotropic helical and non-helical turbulence and its extension to the rotating case. ${ }^{33}$ The model is based on the eddy damped quasi-normal Markovian (EDQNM) closure to compute eddy viscosity and eddy noise and assumes unresolved scales (scales smaller than the cut-off) are isotropic. Both eddy viscosity and noise are computed considering the contribution from the energy and the helicity spectra (see, e.g., Ref. 34 for another subgrid model that takes into account the effect of helicity). The model adapts dynamically to the inertial indices of the resolved energy and helicity spectra, and as a result, it is well suited to study rotating turbulence for which the scaling laws are not well known and may depend on the Rossby number. For a validation of the LES against DNS results, the reader is referred to Refs. 32 and 33.

The subgrid model starts by applying a spectral filter to the equations; this operation consists in truncating all velocity components at wave vectors $\mathbf{k}$ such that $|\mathbf{k}|=k>k_{c}$, where $k_{c}$ is the cut-off wave number. One then models the transfer between the large (resolved) scales and the small (subgrid unresolved) scales of the flow by adding eddy viscosity and eddy noise to the equations for the resolved scales. These are obtained solving the EDQNM equations for estimated energy and helicity spectra in the subgrid range. To this end, an intermediate range is defined, lying between $k_{c}$ and $k_{c}$ (in most cases $k_{c} \prime=k_{c} / 3$ ), where the energy spectrum is assumed to present a power-law behavior possibly followed by an exponential decrease. As an example, for the energy the following expression is used:

$$
E(k, t)=E_{0} k^{-\alpha_{E}} e^{-\delta_{E} k}, \quad k_{c}^{\prime} \leq k<k_{c} .
$$

The coefficients $\alpha_{E}, \delta_{E}$, and $E_{0}$ are computed at each time step doing a mean square fit of the resolved energy spectrum. The spectrum is extrapolated to the unresolved scales using these coefficients, and the EDQNM equations are solved. Then one solves the Navier-Stokes equation (1) with an extra term on the r.h.s. which in spectral space takes the form

$$
-\nu\left(k \mid k_{c}, t\right) k^{2} \mathbf{u}(\mathbf{k}, t)
$$

where $\mathbf{u}(\mathbf{k}, t)$ is the Fourier transform of the velocity field $\mathbf{u}(\mathbf{x}, t),-k^{2}$ is the Laplacian in Fourier space, and $\nu\left(k \mid k_{c}, t\right)$ is an eddy viscosity proportional to the ratio of the so-called absorption terms in EDQNM to the energy (and helicity) spectrum. Eddy noise is added in a similar manner (for more details, see Refs. 32 and 33).

LES runs using this model have a resolution of either $96^{3}$ or $192^{3}$ grid points. A pseudo-spectral method is also used, but without dealiasing, resulting in the maximum wave number $k_{\max }=N / 2$. As in the DNS, an explicit second-order Runge-Kutta method is used to evolve in time.

Parameters for all sets of runs are listed in Table I. DNS runs are labeled with a $\mathrm{D}$, followed by the linear resolution, a letter " $\mathrm{H}$ " if the run has helicity, a letter " $\mathrm{A}$ " if the initial energy spectrum is anisotropic, and the run number. LES runs start with an L, followed by numbers and letters using the same convention as in the DNS.

\section{Initial conditions and definitions}

As mentioned in the Introduction, we are interested in the decay laws obtained in the system depending on properties of the initial conditions and the amount of rotation. In particular, we will vary the initial amount of helicity, the initial energy containing scale (with respect to the largest available scale in the box), the shape of the energy spectrum, and the strength of turbulence and of rotation as controlled by the Reynolds and Rossby numbers, respectively.

Helicity is an ideal invariant of the Navier-Stokes equation which measures the alignment between velocity and vorticity. If zero, the initial conditions are mirror-symmetric, so it also measures the departure from a mirror-symmetric state. We define the net helicity as

$$
H=\langle\mathbf{u} \cdot \omega\rangle,
$$

where the brackets denote spatial average. We also define the relative helicity as

$$
h=\frac{H}{\langle|\mathbf{u}||\omega|\rangle},
$$

which is bounded between -1 and 1 and can be interpreted as the mean cosine of the angle between the velocity and the vorticity.

To control the net amount of relative helicity in the initial conditions, we consider three different flows: a superposition of Taylor-Green (TG) vortices, ${ }^{35}$

$$
\begin{aligned}
\mathbf{u}_{T G}= & U \sin \left(k_{0} x\right) \cos \left(k_{0} y\right) \cos \left(k_{0} z\right) \hat{x} \\
& -U \cos \left(k_{0} x\right) \sin \left(k_{0} y\right) \cos \left(k_{0} z\right) \hat{y},
\end{aligned}
$$

a superposition of Arn'old-Beltrami-Childress (ABC) flows, ${ }^{36}$

$$
\begin{aligned}
\mathbf{u}_{A B C}= & {\left[B \cos \left(k_{0} y\right)+A \sin \left(k_{0} z\right)\right] \hat{x} } \\
& +\left[C \cos \left(k_{0} z\right)+A \sin \left(k_{0} x\right)\right] \hat{y} \\
& +\left[A \cos \left(k_{0} x\right)+B \sin \left(k_{0} y\right)\right] \hat{z}
\end{aligned}
$$

(with $A=0.9, B=1.1$, and $C=1$ ), and a superposition of Fourier modes with random phases (RND) in which we use the algorithm described in Ref. 37 to control the relative helicity. In each case, the flows are superposed in a range of wave numbers as described in Table I and with global amplitudes for each wave number to give the desired slope in the initial energy spectrum. 
TABLE I. Parameters used in the simulations: kinematic viscosity $\nu$, rotation rate $\Omega$, Reynolds number $R e$, Rossby number $R o$, micro-Rossby number $R o^{\omega}$, initial relative helicity $h$, relative helicity at the time of maximum dissipation $h^{*}$, and time of maximum dissipation $t^{*}$. The values of $R e, R o$, and $R o^{\omega}$ are always given at $t^{*}$. The last column succinctly describes the initial energy spectrum $E(k)$ : the power law followed by the spectrum, the range of scales where this power law is satisfied, and the flow (TG for Taylor-Green, ABC for Arn'old-Beltrami-Childress, and RND for random).

\begin{tabular}{|c|c|c|c|c|c|c|c|c|c|}
\hline Run & $\nu$ & $\Omega$ & $\operatorname{Re}$ & Ro & $R o^{\omega}$ & $h$ & $h^{*}$ & $t^{*}$ & Initial $E(k)$ \\
\hline D256-1 & $1.5 \times 10^{-3}$ & 0 & 450 & $\infty$ & $\infty$ & 0 & $9 \times 10^{-10}$ & 1.26 & $k^{-4}(4-14) \mathrm{TG}$ \\
\hline D256-2 & $1.5 \times 10^{-3}$ & 4 & 550 & 0.12 & 1.28 & 0 & $-1 \times 10^{-8}$ & 1.06 & $k^{-4}(4-14) \mathrm{TG}$ \\
\hline $\mathrm{D} 256 \mathrm{H}-1$ & $1.5 \times 10^{-3}$ & 0 & 600 & $\infty$ & $\infty$ & 0.95 & 0.34 & 2.28 & $k^{-4}(4-14) \mathrm{ABC}$ \\
\hline D256H-2 & $1.5 \times 10^{-3}$ & 4 & 830 & 0.08 & 0.80 & 0.95 & 0.65 & 2.25 & $k^{-4}(4-14) \mathrm{ABC}$ \\
\hline D512-1 & $7 \times 10^{-4}$ & 4 & 1100 & 0.12 & 1.82 & 0 & $7 \times 10^{-9}$ & 0.88 & $k^{-4}(4-14) \mathrm{TG}$ \\
\hline D512-2 & $8.5 \times 10^{-4}$ & 0 & 420 & $\infty$ & $\infty$ & $8 \times 10^{-5}$ & $8 \times 10^{-4}$ & 0.60 & $k^{4}(1-14) \mathrm{RND}$ \\
\hline D512-3 & $8.5 \times 10^{-4}$ & 10 & 450 & 0.10 & 0.95 & $4 \times 10^{-3}$ & $4 \times 10^{-3}$ & 0.70 & $k^{4}(1-14) \mathrm{RND}$ \\
\hline D512H-1 & $7 \times 10^{-4}$ & 4 & 1750 & 0.08 & 1.15 & 0.95 & 0.44 & 1.70 & $k^{-4}(4-14) \mathrm{ABC}$ \\
\hline D512H-2 & $8 \times 10^{-4}$ & 0 & 440 & $\infty$ & $\infty$ & 0.90 & 0.38 & 0.94 & $k^{4}(1-14) \mathrm{RND}$ \\
\hline D512H-3 & $8 \times 10^{-4}$ & 10 & 530 & 0.07 & 0.70 & 0.99 & 0.5 & 1.50 & $k^{4}(1-14) \mathrm{ABC}$ \\
\hline L96-1 & $8.5 \times 10^{-4}$ & 0 & 550 & $\infty$ & $\infty$ & 0.03 & 0.02 & 0.30 & $k^{4}(1-14) \mathrm{RND}$ \\
\hline L96-2 & $8.5 \times 10^{-4}$ & 2 & 540 & 0.42 & 2.90 & -0.03 & -0.02 & 0.30 & $k^{4}(1-14) \mathrm{RND}$ \\
\hline L96-3 & $8.5 \times 10^{-4}$ & 4 & 540 & 0.21 & 1.45 & -0.03 & -0.02 & 0.30 & $k^{4}(1-14) \mathrm{RND}$ \\
\hline L96-4 & $8.5 \times 10^{-4}$ & 6 & 550 & 0.14 & 0.95 & -0.03 & -0.02 & 0.30 & $k^{4}(1-14) \mathrm{RND}$ \\
\hline L96-5 & $8.5 \times 10^{-4}$ & 8 & 550 & 0.11 & 0.73 & -0.03 & -0.02 & 0.30 & $k^{4}(1-14)$ RND \\
\hline L96-6 & $8.5 \times 10^{-4}$ & 10 & 530 & 0.08 & 0.65 & 0.03 & 0.02 & 0.30 & $k^{4}(1-14) \mathrm{RND}$ \\
\hline L96H-1 & $8 \times 10^{-4}$ & 0 & 500 & $\infty$ & $\infty$ & 0.90 & 0.51 & 0.70 & $k^{4}(1-14) \mathrm{RND}$ \\
\hline L96H-2 & $8.5 \times 10^{-4}$ & 10 & 540 & 0.08 & 0.63 & 0.90 & 0.70 & 0.70 & $k^{4}(1-14) \mathrm{RND}$ \\
\hline L96H-3 & $8.5 \times 10^{-4}$ & 10 & 490 & 0.08 & 0.60 & 0.99 & 0.80 & 1.15 & $k^{4}(1-14) \mathrm{ABC}$ \\
\hline L192-1 & $2 \times 10^{-4}$ & 0 & 1200 & $\infty$ & $\infty$ & $-7 \times 10^{-3}$ & $-6 \times 10^{-3}$ & 0.10 & $k^{4}(1-30) \mathrm{RND}$ \\
\hline L192-2 & $2 \times 10^{-4}$ & 10 & 1100 & 0.22 & 1.65 & $-7 \times 10^{-3}$ & $-6 \times 10^{-3}$ & 0.13 & $k^{4}(1-30)$ RND \\
\hline L192H-1 & $2 \times 10^{-4}$ & 0 & 950 & $\infty$ & $\infty$ & 0.90 & 0.60 & 0.30 & $k^{4}(1-30) \mathrm{RND}$ \\
\hline L192H-2 & $2 \times 10^{-4}$ & 10 & 1000 & 0.20 & 1.60 & 0.94 & 0.71 & 0.38 & $k^{4}(1-30) \mathrm{ABC}$ \\
\hline L192HA-1 & $2 \times 10^{-4}$ & 10 & 1200 & 0.16 & 1.40 & 0.90 & 0.56 & 0.50 & $k^{4}(1-25) \mathrm{RND}$ \\
\hline L192HA-2 & $2 \times 10^{-4}$ & 10 & 1300 & 0.14 & 1.35 & 0.90 & 0.59 & 0.46 & $k^{4}(1-25) \mathrm{RND}$ \\
\hline L192HA-3 & $2 \times 10^{-4}$ & 10 & 1300 & 0.15 & 1.35 & 0.90 & 0.58 & 0.45 & $k^{4}(1-25) \mathrm{RND}$ \\
\hline
\end{tabular}

The TG vortex is non-helical $(h=0)$ and has no energy in the $k_{z}=0$ modes, whose amplification in the rotating cases (see below) can thus be attributed only to a bidimensionalization process. The TG vortex was originally motivated as an initial condition which leads to rapid development of small spatial scales and also mimics the von Kármán flow between two counter-rotating disks used in several experiments. ${ }^{38}$ The $\mathrm{ABC}$ flow is an eigenfunction of the curl operator and as a result has maximum helicity $(h= \pm 1$ depending on the sign of $k_{0}$, when only one value of $k_{0}$ is excited), whereas the RND flow allows us to tune the amount of initial relative helicity between -1 and 1 as well as the initial anisotropy.

When generating the flows, two different initial energy spectra were considered. To study initial conditions with characteristic length close to the size of the computational domain, a spectrum $E(k) \sim k^{-4}$ for $k \in[4,14]$ (followed by exponential decay) was imposed. To study initial conditions with length smaller than the domain size, we imposed a spectrum $E(k) \sim k^{4}$ for $k \in[1,14],[1,25]$, or [1, 30] (also followed by exponential decay). In the latter case, the characteristic length can grow in time as the spectrum peaks around $k=14,25$, or 30 . This allows us to mimic (at least for a finite time before the characteristic length reaches the domain size) the decay of unbounded flows. The characteristic length will be associated in the following with the flow integral scale, which is defined as

$$
L=2 \pi \frac{\sum_{k} k^{-1} E(k)}{\sum_{k} E(k)}
$$

where $E(k)$ is the isotropic energy spectrum.

Simulations in Table I are also characterized by different Reynolds and Rossby numbers. The Reynolds and Rossby numbers in the table are defined as

$$
R e=\frac{U L}{\nu},
$$

and

$$
R o=\frac{U}{2 \Omega L},
$$

respectively. Of importance is also the micro-scale Rossby number (see e.g., Ref. 7)

$$
R o^{\omega}=\frac{\omega}{2 \Omega},
$$

which can be interpreted as the ratio of the convective to the Coriolis acceleration at the Taylor scale. The Rossby number $R o$ must be small enough for rotation to affect the turbulence, while the micro-Rossby number $R o^{\omega}$ must be larger than one for scrambling effects of inertial waves not to completely damp the nonlinear term, which would lead to pure 
exponential viscous energy decay. ${ }^{5}$ In all runs in Table I, Ro and $R o^{\omega}$ are one order of magnitude apart at the time of maximum enstrophy $t^{*}$, and this interval is roughly preserved throughout the simulations.

Here and in the following, the isotropic energy spectrum is defined by averaging in Fourier space over spherical shells

$$
E(k, t)=\frac{1}{2} \sum_{k \leq|\mathbf{k}|<k+1} \mathbf{u}^{*}(\mathbf{k}, t) \cdot \mathbf{u}(\mathbf{k}, t),
$$

where $\mathbf{u}(\mathbf{k}, t)$ is the Fourier transform of the velocity field, and the asterisk denotes complex conjugate. Other two spectra can also be used to characterize anisotropy.

On the one hand, the so-called "reduced" energy spectra $E\left(k_{\perp}\right)$ and $E\left(k_{\|}\right)$are defined averaging in Fourier space over cylinders and planes, respectively. More specifically, the reduced energy spectra as a function of wave numbers $k_{\perp}$ with $\mathbf{k}_{\perp}=\left(k_{x}, k_{y}, 0\right)$ and $k_{\|}$with $\mathbf{k}_{\|}=\left(0,0, k_{z}\right)$ are defined by computing the sum above over all modes in the cylindrical shells $k_{\perp} \leq\left|\mathbf{k}_{\perp}\right|<k_{\perp}+1$ and over planes $k_{\|} \leq\left|\mathbf{k}_{\|}\right|<k_{\|}$ +1 , respectively (isotropic and reduced spectra for the helicity are defined in the same way). From the reduced spectra, perpendicular and parallel integral scales can be defined; e.g., for the perpendicular direction,

$$
L_{\perp}=2 \pi \frac{\sum_{k_{\perp}} k_{\perp}^{-1} E\left(k_{\perp}\right)}{\sum_{k_{\perp}} E\left(k_{\perp}\right)} .
$$

On the other hand, more information of the spectral anisotropy can be obtained studying the axisymmetric energy spectrum $e\left(k_{\|}, k_{\perp}\right)$ (see, e.g., Refs. 3 and 5). Assuming the flow is axisymmetric, the three-dimensional spectrum can be integrated around the axis of rotation to obtain a spectrum that depends only on $k_{\|}$and $k_{\perp}$, which relates to the reduced energy spectra as follows:

$$
E\left(k_{\|}\right)=\sum_{k_{\perp}} e\left(k_{\|}, k_{\perp}\right),
$$

and

$$
E\left(k_{\perp}\right)=\sum_{k_{\|}} e\left(k_{\|}, k_{\perp}\right)
$$

\section{TIME EVOLUTION: PHENOMENOLOGY}

We present now phenomenological arguments that will become handy to understand the different decay rates that are observed in our simulations as well as in previous studies. Some of the arguments are well known, while others are new, and we quote previous derivations when needed.

\section{A. Non-rotating flows}

\section{Bounded}

From the energy balance equation

$$
\frac{d E}{d t} \sim \epsilon,
$$

where $\epsilon$ is the energy dissipation rate, Kolmogorov phenomenology leads to

$$
\frac{d E}{d t} \sim \frac{E^{3 / 2}}{L}
$$

where $E=E(t) \sim k E(k)$ and $L$ is an energy containing length scale. For bounded flows where $L \sim L_{0}\left(L_{0}\right.$ is the size of the box), Eq. (18) becomes $d E / d t \sim E^{3 / 2} / L_{0}$, resulting in the self-similar decay ${ }^{18,39,40}$

$$
E(t) \sim t^{-2}
$$

\section{Unbounded}

In unbounded flows, a similarity solution of Eq. (18) requires some knowledge of the behavior of the energy containing scale $L$, which is in turn related to the evolution of $E(k)$ for low wave numbers. In the case of an initial large scale spectrum $\sim k^{4}$, the quasi-invariance of Loitsyanski's integral $I$ (see Refs. 21 and 41) leads, on dimensional grounds, to $I \sim L^{5} E$, and replacing in Eq. (18), we get Kolmogorov's result $^{20}$

$$
E(t) \sim t^{-10 / 7}
$$

A different decay law is obtained if an initial $\sim k^{2}$ spectrum is assumed for low wave numbers. ${ }^{22,23}$ In the following, we will consider only the bounded or the $\sim k^{4}$ unbounded cases.

\section{B. Rotating flows: Isotropic arguments}

\section{Bounded}

In the case of solid-body rotation without net helicity, a spectra $E(k) \sim \epsilon^{1 / 2} \Omega^{1 / 2} k^{-2}$ is often assumed at small scales (i.e., wave numbers larger than the integral wave number)..$^{9,42-45}$ Replacing in the balance equation, this spectrum leads to

$$
\frac{d E}{d t} \sim \frac{E^{2}}{L^{2} \Omega}
$$

For bounded flows, $L \sim L_{o}$ and we get ${ }^{13,44,46}$

$$
E(t) \sim t^{-1}
$$

In helical rotating flows, the small-scale energy spectrum takes a different form. The direct transfer is dominated by the helicity cascade. In this case, we can write the helicity flux as $\delta \sim h_{l} /\left(\Omega \tau_{l}^{2}\right)$, where $h_{l}$ is the helicity at the scale $l$, and $\tau_{l}$ the eddy turnover time. ${ }^{11}$ Constancy of $\delta$ leads to small scale spectra $E(k) \sim k^{-n}$ and $H(k) \sim k^{n-4}$, where the case of maximum helicity is obtained for $n=5 / 2 .^{11}$ Further use of dimensional analysis leads to $E(k) \sim \epsilon^{1 / 4} \Omega^{5 / 4} k^{-5 / 2}$ for the energy spectrum in terms of the energy dissipation rate, and replacing in the balance equation, we get

$$
\frac{d E}{d t} \sim \frac{E^{4}}{L^{6} \Omega^{5}}
$$


For $L \sim L_{o}$, then ${ }^{19}$

$$
E(t) \sim t^{-1 / 3}
$$

\section{Unbounded}

For non-helical unbounded flows with $E(k) \sim k^{4}$ at small wave numbers, we can again make use of the constancy of $I$ in Eq. (21), leading to ${ }^{25}$

$$
E(t) \sim t^{-5 / 7}
$$

For helical flows, assuming that $I$ remains constant in Eq. (23), we obtain ${ }^{47}$

$$
E(t) \sim t^{-5 / 21}
$$

\section{Rotating flows: Anisotropic arguments}

The decay laws obtained for rotating flows in Eqs. (22), (24), and (25) have been reported in experiments or in simulations. ${ }^{12,13,19,24}$ However, the analysis above is based on the isotropic energy spectrum and on the quasi-invariance of the isotropic Loitsyanski integral. For an anisotropic flow, other quantities are expected to be quasi-invariants during the decay instead. ${ }^{41,48,49}$

Rotating flows tend to become quasi-2D, and the assumption of an axisymmetric energy spectrum seems natural considering the symmetries of the problem. If there is no dependence on wave numbers on the parallel direction, the energy spectrum for small values of $k_{\perp}$ can be expanded as (see, e.g., Ref. 50)

$$
E\left(k_{\perp}\right) \approx L k_{\perp}^{-1}+K k_{\perp}+I_{2 D} k_{\perp}^{3}+\cdots .
$$

We will be interested in the following coefficients:

$$
K=\int\left\langle\mathbf{u} \cdot \mathbf{u}^{\prime}\right\rangle r d r
$$

and

$$
I_{2 D}=\int\left\langle\mathbf{u} \cdot \mathbf{u}^{\prime}\right\rangle r^{3} d r,
$$

where $\left\langle\mathbf{u} \cdot \mathbf{u}^{\prime}\right\rangle$ is the two-point correlation function for the spatial increments $r$ perpendicular to the rotation axis. If the correlation function decays fast enough for large values of $r{ }^{48}$ these quantities can be expected to be quasi-invariants during the decay, respectively, for initial large-scale energy spectra $\sim k_{\perp}$ and $\sim k_{\perp}^{3}$, and in the same way $I$ is quasi-conserved during the decay of isotropic flows with an initial large-scale $\sim k^{4}$ energy spectrum. A detailed proof of the conservation of $K$ for rotating flows can be found in Ref. 49; it is a direct consequence of the conservation of linear momentum in the direction parallel to the rotation axis. It is worth pointing out that these quantities were also shown to be conserved in other systems: proofs of the conservation of $K$ and $I_{2 D}$ for quasigeostrophic flows can be found in Ref. 50 . In practice, these quantities are only approximately conserved in numerical simulations, see e.g., the approximate constancy of $I_{2 D}$ and $K$ reported for rotating flows in Ref. 47.
As per virtue of the decay the Rossby number decreases with time, we will further assume for our phenomenological analysis that 2D and 3D modes are only weakly coupled and write equations for the energy in the $2 \mathrm{D}$ modes, $E_{2 D}$. In the non-helical case, if $K$ remains approximately constant with $K \sim E_{2 D} L_{\perp}^{2} L_{0 \|}$ (where $L_{0 \|}$ is the size of the box in the direction parallel to $\boldsymbol{\Omega}$ ), then Eq. (21) for the $2 \mathrm{D}$ modes becomes

$$
\frac{d E_{2 D}}{d t} \sim \frac{E_{2 D}^{3} L_{0 \|}}{K \Omega},
$$

which leads to a decay

$$
E_{2 D}(t) \sim t^{-1 / 2}
$$

Alternatively, constancy of $I_{2 D} \sim E_{2 D} L_{\perp}^{4} L_{0 \|}$ in Eq. (21) for the $2 \mathrm{D}$ modes leads to

$$
\frac{d E_{2 D}}{d t} \sim \frac{E_{2 D}^{5 / 2} L_{0 \|}^{1 / 2}}{I_{2 D}^{1 / 2} \Omega}
$$

and

$$
E_{2 D}(t) \sim t^{-2 / 3}
$$

The same arguments can be extended to the helical rotating case using Eq. (23). If constancy of $K$ is assumed, we get

$$
\frac{d E_{2 D}}{d t} \sim \frac{E_{2 D}^{7} L_{0 \|}^{3}}{\Omega^{5} K^{3}}
$$

and

$$
E(t) \sim t^{-1 / 6}
$$

Finally, constancy of $I_{2 D}$ leads to

$$
\frac{d E_{2 D}}{d t} \sim \frac{E_{2 D}^{11 / 2} L_{0 \|}^{3 / 2}}{I_{2 D}^{3 / 2} \Omega^{5}}
$$

and

$$
E(t) \sim t^{-2 / 9}
$$

These decay laws will be important to analyze the evolution of the energy in the simulations discussed in the next section.

\section{Enstrophy decay}

From any of the previous energy decay laws, one can also compute laws for the enstrophy decay $\Omega(t)=\left\langle\omega^{2}\right\rangle / 2$ using the isotropic energy balance equation and replacing $\epsilon=\nu \Omega(t)$, which results in $\Omega(t)=\nu^{-1} d E / d t$. From this equation, for every solution for which the energy decays as $E(t) \sim t^{\alpha}$, the enstrophy decay results

$$
\Omega(t) \sim t^{\alpha-1} .
$$

Although rotating flows are anisotropic, the enstrophy is predominantly a small-scale magnitude and we will see that this isotropic argument gives good agreement with the numerical results for rotating and non-rotating flows. Since helicity is 
related with the energy and the enstrophy only through a Schwartz inequality, no simple decay laws can be derived in its case using these phenomenological arguments.

\section{TIME EVOLUTION: NUMERICAL RESULTS}

We present here the results for the energy, enstrophy, and helicity decay obtained in the numerical simulations listed in Table I, classifying them as rotating or non-rotating, bounded or unbounded (in the sense that the initial integral scale is smaller than the size of the box), and helical or non-helical.

Concerning the terminology of "bounded" and "unbounded" used to describe the numerical simulations, it is important to note that confinement effects in a rotating flow go beyond a saturation of the integral scale when it grows to the box size. Confinement also selects a discrete set of inertial waves which are normal modes of the domain, and boundaries can introduce dissipation through Ekman layers. The latter effect is not present in our numerical simulations with periodic boundary conditions. Finally, it was shown in Ref. 51 (see also Ref. 52) that the small number of Fourier modes available in the shells with wave number $k \approx 1$ gives rise to poor representation of isotropy and of the integral scale in runs for which the integral scale approaches $1 / 5$ of the box size. As a result, the "bounded" runs are here only briefly considered to study the time evolution of global quantities (energy, enstrophy, and helicity) and to compare with the prediction obtained in the corresponding cases in the phenomenological analysis.

\section{A. Non-rotating flows}

Numerical results for non-rotating, bounded, and unbounded flows are shown in Fig. 1. In the unbounded case (runs with an initial energy spectrum $\sim k^{4}$ peaking at $k=14$ in the DNS and $96^{3}$ LES, and peaking at $k=30$ in the $192^{3}$ LES), the runs show a decay for the energy close to $\sim t^{-10 / 7}$, independently of the presence of helicity or not (note the runs also span a range of Reynolds numbers from $R e \approx 420$ to 1200 ). The decay is consistent with the prediction given by Eq. (20) for an initial $\sim k^{4}$ energy spectrum.

The enstrophy decay is also consistent with this law, as expressed by Eq. (38), decaying close to $\sim t^{-17 / 7}$ in all cases. In the absence of rotation, helicity only delays the onset of the self-similar decay by retarding the time when the maximum of enstrophy takes place, as already reported in Refs. 27 and 53. This is more clearly seen in the DNS, see, e.g., the time of the peak of enstrophy for runs D512-3 and D512H-3 in Fig. 1(b). Finally, in the helical runs, helicity seems to decay as the enstrophy, just slightly slower than the $\sim t^{-17 / 7}$ law.

Similar results are observed for bounded flows, i.e., for initial conditions with the initial energy containing scale close to the size of the box (runs with a $\sim k^{-4}$ spectrum from $k=4$ to 14 , peaking at $k=4)$. In this case, all runs are consistent with a $\sim t^{-2}$ decay for the energy (see the insets of Fig. 1) in agreement with Eq. (19), and a decay for the enstrophy close to $\sim t^{-3}$ in agreement with Eq. (38). In the helical runs, helicity decays again slightly slower than the enstrophy but close to the $\sim t^{-3}$ power law.

\section{B. Rotating flows}

\section{Global quantities}

As rotation is increased, the simulations show a shallower power law in the energy decay. As an illustration, Fig. 2 shows the energy decay rate in simulations of unbounded non-helical flows with increasing rotation rate $\Omega$. As reported in previous numerical simulations ${ }^{24}$ and experiments, ${ }^{12,13}$ as
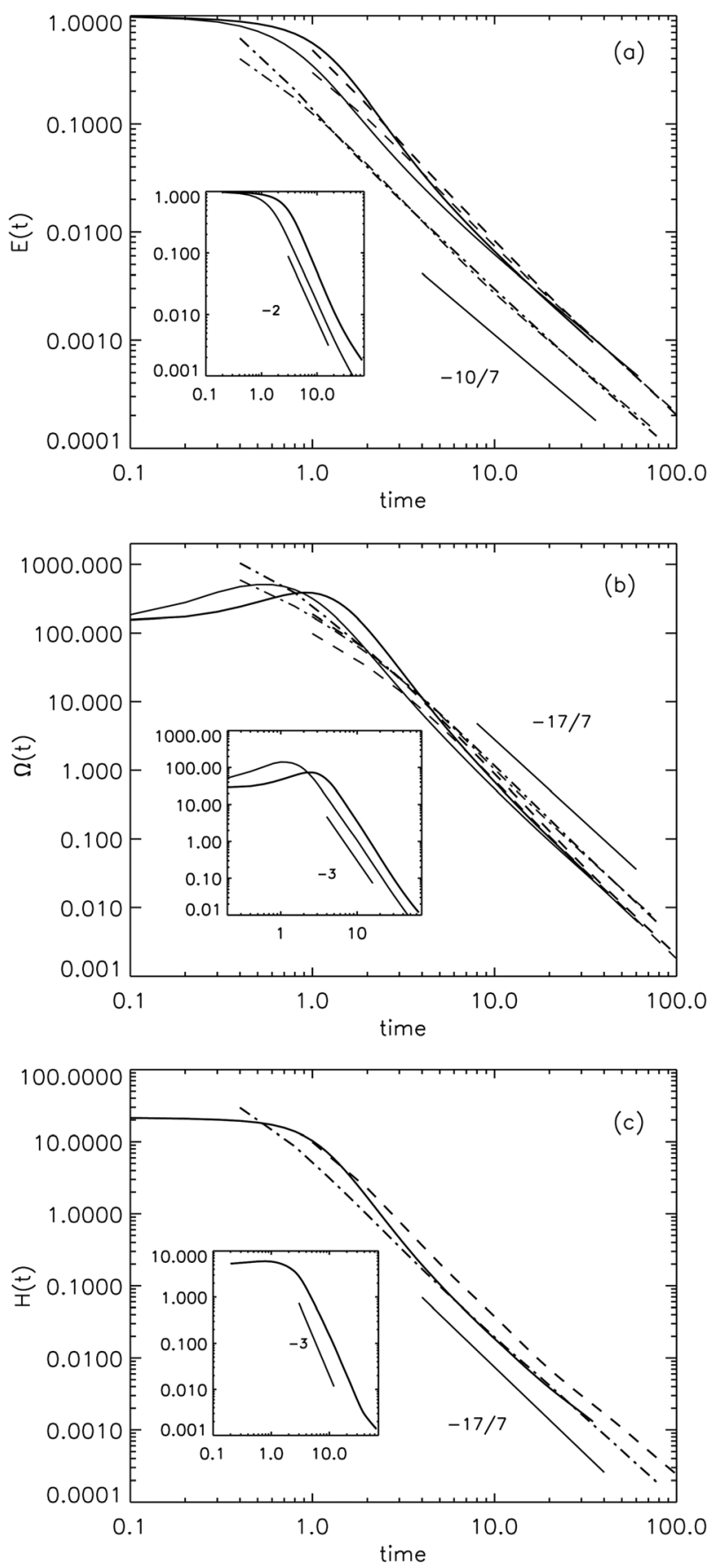

FIG. 1. (a) Energy decay for non-rotating unbounded runs. Non-helical runs D512-2 (solid), L96-1 (dashed), and L192-1 (dashed-dotted) and helical runs D512H-2 (solid, thick), L96H-1 (dashed, thick), and L192H-1 (dasheddotted, thick) are shown. A $-10 / 7$ slope is shown as a reference. The inset shows the energy decay for non-rotating bounded runs D256-1 (solid) and D256H-1 (solid, thick). (b) Enstrophy decay for the same runs, with a $-17 / 7$ slope shown as a reference. The inset shows the enstrophy decay in the bounded runs. (c) Helicity decay in the unbounded helical runs of (a); the inset shows the helicity decay for the bounded helical run D256H-1. 


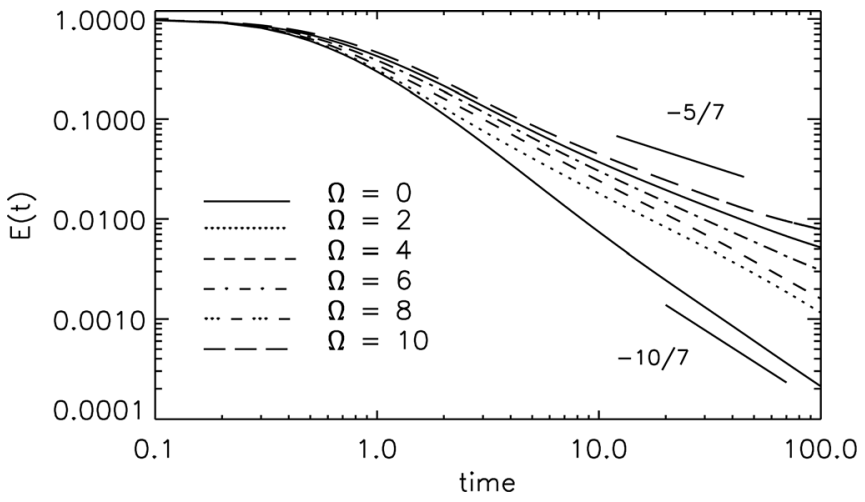

FIG. 2. Energy decay for different values of $\Omega$ from 0 to 10 for unbounded, non-helical runs L96-1, L96-2, L96-3, L96-4, L96-5, and L96-6. The decay becomes slower with increasing rotation rate. We also show $t^{-10 / 7}$ and $t^{-5 / 7}$ laws as references.

$\Omega$ increases the decay slows down until reaching a saturated decay for $R o \approx 0.1$. We will focus in the following in simulations with Rossby number small enough to observe this saturated decay, although not so small that the rotation quenches all non-linear interactions giving only exponential decay. A detailed study of the transition between the non-rotating and rotating cases can be found in Ref. 12.

Figure 3 shows the energy, enstrophy, and helicity decay in simulations of rotating flows with and without helicity, in the unbounded and bounded cases (the latter in the insets). The energy decay in the unbounded non-helical runs (thin lines in Fig. 3) is slightly steeper than what Eq. (25) predicts $\left(E \sim t^{-5 / 7}\right)$. A better agreement is observed for the enstrophy, which is closer to a $\sim t^{-12 / 7}$ law. As will be shown next, the agreement between the phenomenological arguments for the energy and the simulations is improved if the decay of 2D and 3D modes is considered separately.

Alternatively, the unbounded helical runs (thick lines in Fig. 3) show for the energy a $\sim t^{-1 / 3}$ decay or steeper (although shallower than $\sim t^{-5 / 7}$ ). Runs with $\mathrm{ABC}$ initial conditions tend to develop a clearer power law decay and to be closer to a $\sim t^{-1 / 3}$ decay than runs with random helical modes. Again, these differences can be explained considering the decay of 2D and 3D modes, as well as the effect of anisotropy in the initial conditions which is specially relevant for this particular case. The enstrophy and helicity show a decay close to $\sim t^{-12 / 7}$. Note that in the presence of rotation, helicity not only slows down the occurrence of the peak of enstrophy as already reported in Ref. 27, but it also changes the energy decay after this peak. The enstrophy decay is not affected by the presence of helicity.

Overall, the case of constrained runs shows a similar scenario, with a significant slow down of the decay rates in the presence of rotation, and with an extra slow down of energy decay in the presence of helicity (see the insets in Fig. 3). Rotating non-helical flows are close to $E(t) \sim t^{-1}, \Omega(t) \sim$ $t^{-2}$, and $H(t) \sim t^{-2}$, while helical flows in this case display a shallower decay in the energy consistent with $E \sim t^{-1 / 3}$ as predicted by the phenomenological arguments that take into account the effect of helicity in the energy spectrum of rotating turbulence. As in the unbounded case, the presence of helicity does not affect the decay rate of enstrophy.

\section{Anisotropic global quantities}

Although the impact of rotation and helicity in the energy decay is clear, the predictions given by the isotropic phenomenological arguments in Sec. B do not coincide in all cases with the results from the simulations. This can be ascribed to the fact that these arguments assume an isotropic energy scaling, while rotation breaks down isotropy, making spectral energy distribution become axisymmetric with the preferred direction along the axis of rotation. Two-dimensionalization of the flow has already been reported during the decay, ${ }^{24,27}$ as
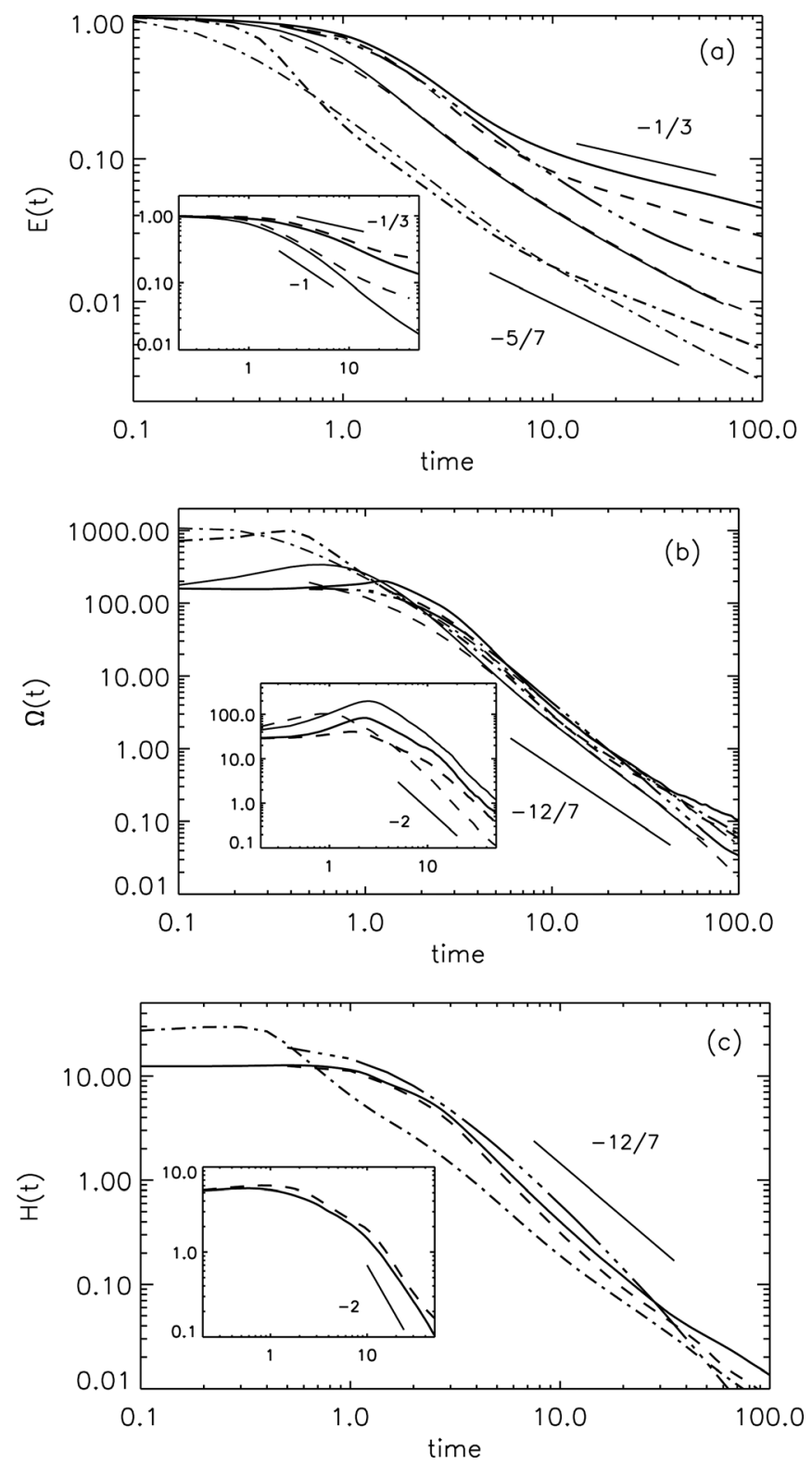

FIG. 3. (a) Energy decay for rotating unbounded runs $(\Omega=10)$. Non-helical runs D512-3 (solid), L96-6 (dashed), and L192-2 (dashed-dotted) and helical runs D512H-3 (solid, thick), L96H-3 (dashed, thick), L96H-2 (dashed-tripedotted, thick), and L192H-2 (dashed-dotted, thick) are shown. At late times, the non-helical runs decay slightly faster than $t^{-5 / 7}$, while the helical runs are close to a $-1 / 3$ decay. The inset shows bounded non-helical runs D256-2 (solid) and D512-1 (dashed) and helical runs D256H-2 (solid, thick) and D512H-1 (dashed, thick). (b) Enstrophy decay for the same runs, with a $12 / 7$ slope shown as a reference. The inset shows the enstrophy decay in the bounded runs. (c) Helicity decay in the unbounded helical runs; bounded helical runs are shown in the inset. 
well as weak coupling of 2D and 3D modes ${ }^{6,15}$ for very small Rossby numbers. Based on this, we discriminate between the energy contained in $3 \mathrm{D}$ modes with $k_{z} \neq 0\left(E_{3 D}\right)$ and the energy in slow 2D modes with $k_{z}=0\left(E_{2 D}\right)$. At this point, it is
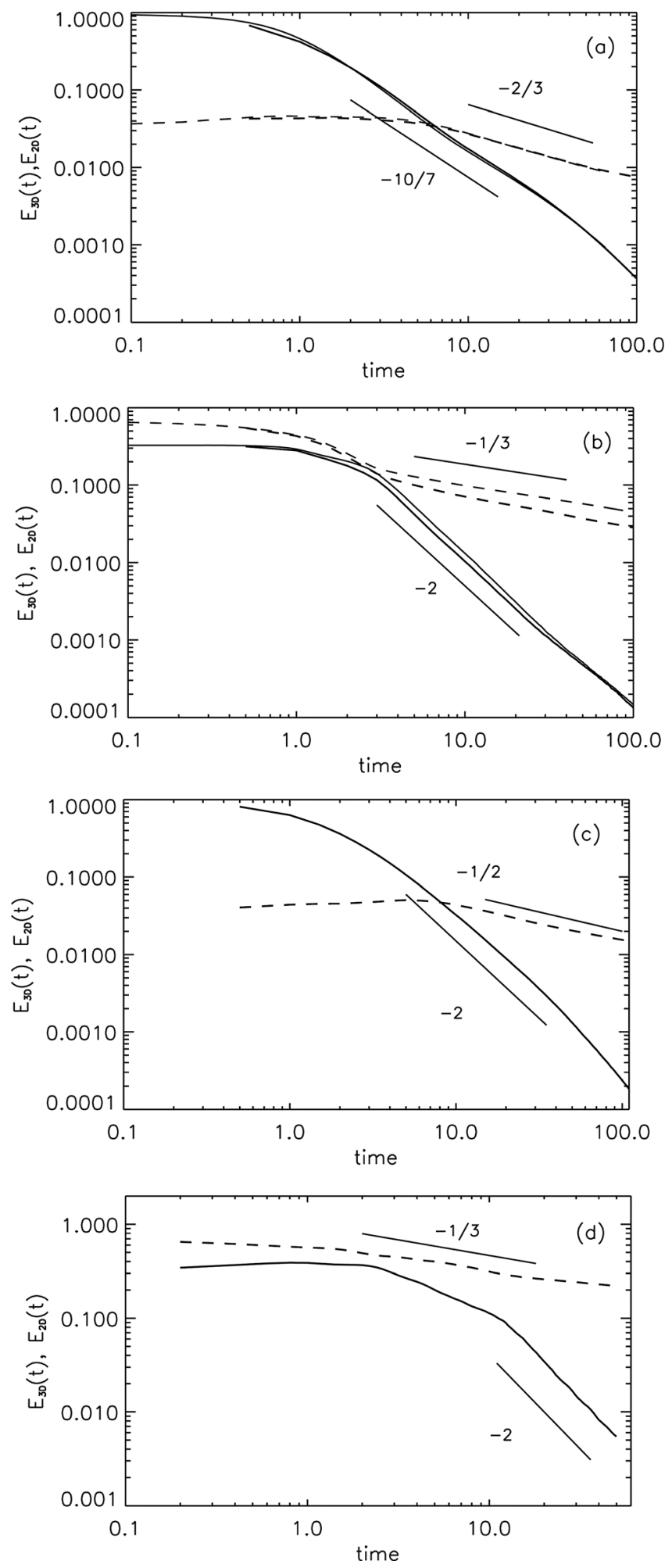

FIG. 4. Energy decay for $E_{3 D}$ (solid) and $E_{2 D}$ (dashed) for runs with rotation. (a) Unbounded non-helical D512-3 (thin) and L96-6 (thick); $E_{3 D} \sim t^{-10 / 7}$ and $E_{2 D} \sim t^{-2 / 3}$ decays are indicated. (b) Unbounded helical with $\mathrm{ABC}$ initial conditions D512H-3 (thin) and L96H-3 (thick); $E_{2 D}$ is close to $t^{-1 / 3}$. (c) Unbounded helical with random initial conditions L96H-2; $E_{2 D}$ is close to $t^{-1 / 2}$. (d) Bounded helical with ABC initial conditions D512H-1. important to point out that as the energy decays in the simulations, the Rossby number also monotonically decreases with time. As a rule, the Rossby numbers decrease by one order of magnitude in the first turnover times (from the values listed in Table I, which correspond to the time of maximum dissipation) and another order of magnitude at $t \approx 100$.

In Fig. 4, we show $E_{3 D}$ and $E_{2 D}$ as a function of time for several runs. In each and every case, we can clearly identify distinct behaviors for the 2D and 3D energies, obeying different power-law decays. On the one hand, $E_{3 D}$ always shows a decay close to some power law expected for a (bounded or unbounded) non-rotating case, with the unbounded non-helical runs having $E_{3 D} \sim t^{-10 / 7}$ in agreement with Eq. (20) as illustrated in Fig. 4(a), and with most helical runs (bounded and unbounded) with $E_{3 D} \sim t^{-2}$ in agreement with Eq. (19) (which corresponds to the bounded non-rotating decay) as illustrated in Figs. 4(b)-4(d). On the other hand, $E_{2 D}$ follows power laws close to the ones predicted by Eqs. (31)-(37). The unbounded non-helical runs are compatible with $E_{2 D} \sim t^{-2 / 3}$, and the helical runs show $\sim t^{-1 / 2}$ or $\sim t^{-1 / 3}$ (note in the helical case, the power laws predicted are for the case of maximum helicity, and for intermediate helicity, the power laws are bounded between the non-helical and the maximally helical values).

The results in Fig. 4 indicate clearer power law decay (and better agreement with phenomenological arguments) is obtained for the separate energy in 2D and 3D modes, than when the total energy is considered (compare, e.g., the extent of the power laws in this figure with the ones in Fig. 3). This is clearer in the non-helical case, where all unbounded runs show a decay consistent with Eq. (33) for the 2D modes (all non-helical runs with random initial conditions have a $\sim k_{\perp}^{3}$ initial energy spectrum, per virtue of the isotropic initial $\sim k^{4}$ spectrum), and with Eq. (20) for the 3D modes. The separate evolution of $E_{3 D}$ and $E_{2 D}$ seems to be independent of the initial ratio of energy in 3D and 2D modes, at least for the range of parameters explored in this work.

To further show the agreement with the phenomenological arguments, the evolution of $I, I_{2 D}$, and $K$ must be considered to verify whether these quantities behave in agreement with the assumptions used to derive the decay laws in Sec.

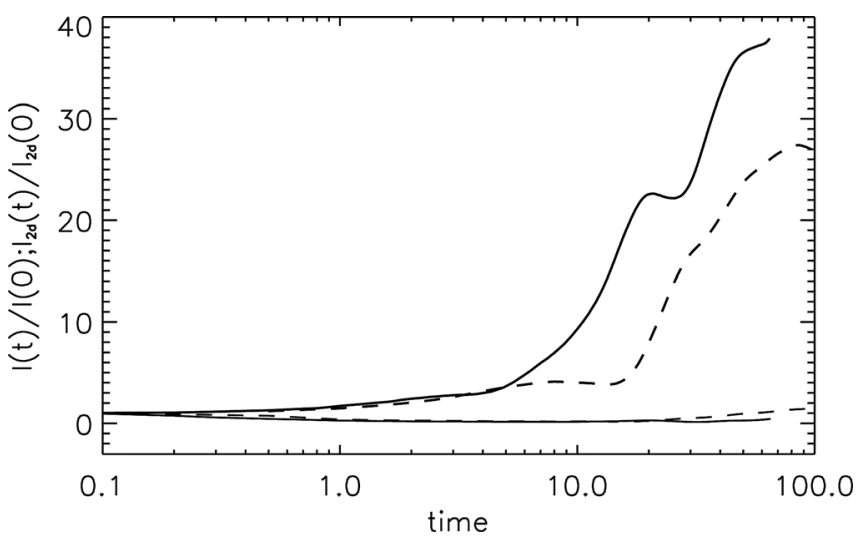

FIG. 5. Evolution of $I / I(0)$ (thick lines) and $I_{2 D} / I_{2 D}(0)$ (thin lines) for runs D512-3 (solid) and L96-6 (dashed). While in both runs $I_{2 D}$ maintains an approximately constant value, $I$ growths monotonically and during the selfsimilar energy decay increases by one order of magnitude. 

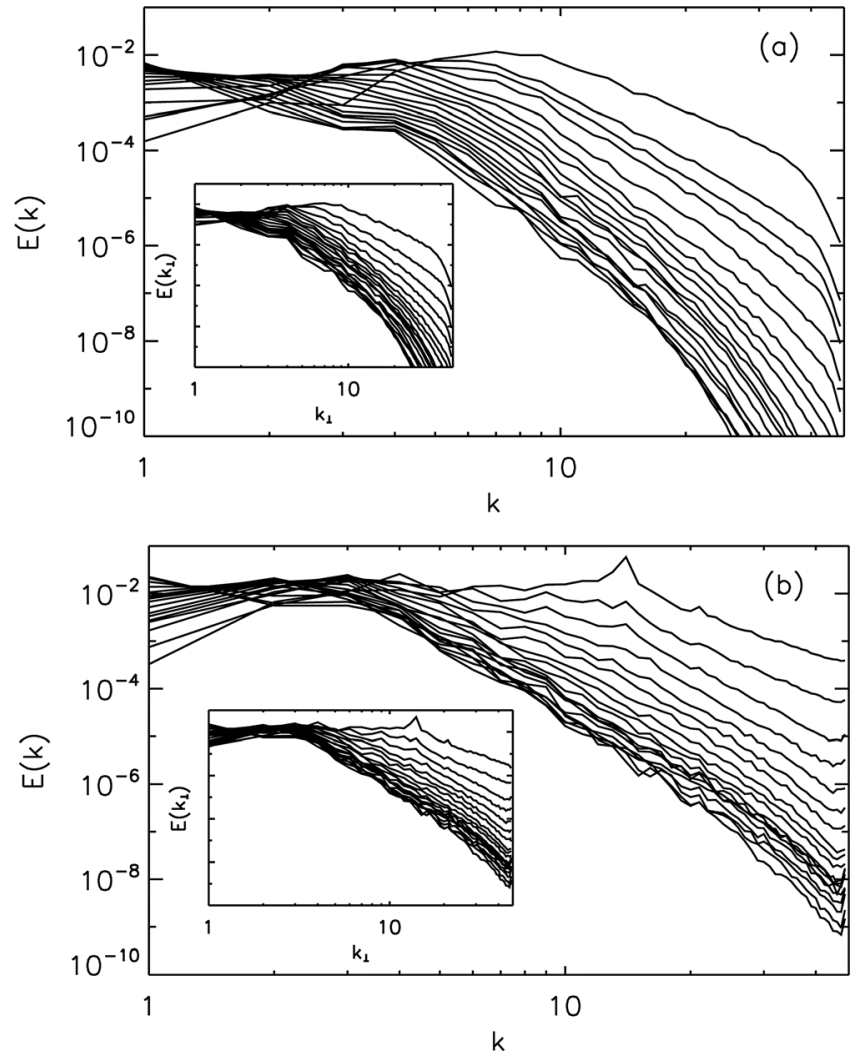

FIG. 6. (a) Evolution of the isotropic energy spectrum $E(k)$ for L96-6 (nonhelical, $\Omega=10$, initial $\sim k^{4}$ spectrum peaking at $k=14$ ) from $t=5$ to $t=100$ with time increments $\Delta t=5$. Inset: reduced perpendicular energy spectrum $E\left(k_{\perp}\right)$ for the same times. (b) Evolution of the isotropic energy spectrum for L96H-2 (helical, $\Omega=10$, initial $\sim k^{4}$ spectrum peaking at $k=14)$ at the same times, with the reduced perpendicular energy spectrum in the inset.
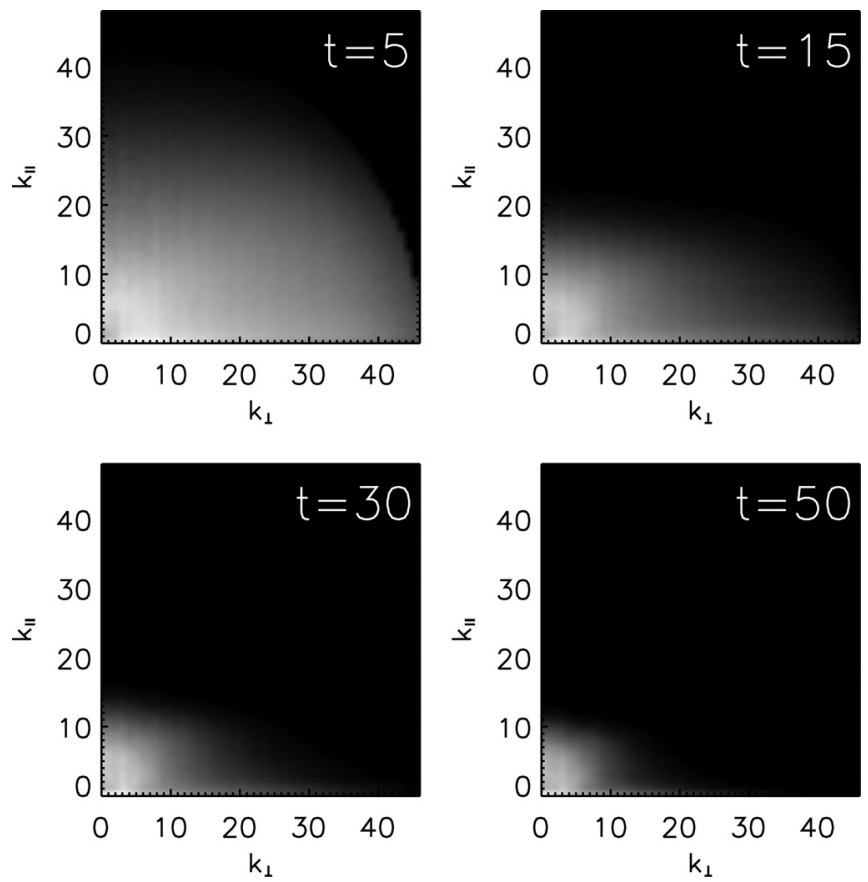

FIG. 7. Axisymmetric energy spectrum $e\left(k_{\|}, k_{\perp}\right) / \sin \theta$ for different times for run L96-6 (non-helical, $\Omega=10$, initial energy spectrum $\sim k^{4}$ peaking at $k=14)$.
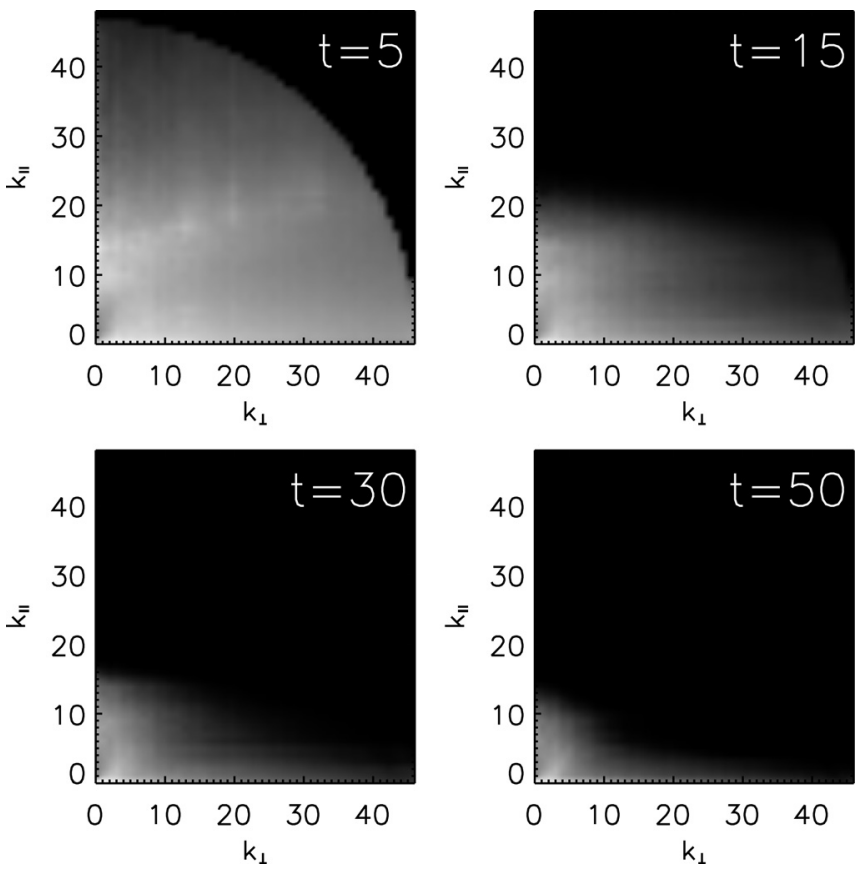

FIG. 8. Axisymmetric energy spectrum $e\left(k_{\|}, k_{\perp}\right) / \sin \theta$ for different times for run $\mathrm{L} 96 \mathrm{H}-3$ (helical with $\mathrm{ABC}$ initial conditions, $\Omega=10$, initial energy spectrum $\sim k^{4}$ peaking at $k=14$ ).

III. Figure 5 shows the time evolution of $I$ and $I_{2 D}$ for two simulations with rotation, normalized by their initial values at $t=0$. In both cases, $I$ grows monotonically in time by a factor of $\approx 50$ during the decay. This fast increase of $I$ is observed in all rotating runs. Meanwhile, $I_{2 D}$ settles and remains approximately constant, fluctuating around its initial value.

The helical runs show more disparity in the time evolution of the $2 \mathrm{D}$ and $3 \mathrm{D}$ energies. Bounded runs show $E_{2 D} \sim t^{-1 / 3}$ and $E_{3 D} \sim t^{-2}$, which agree with the previous scenario where 3D modes decay as in the non-rotating case and slow 2D modes decay according to the anisotropic prediction with rotation (in this case, corresponding to Eqs. (24) and (19), respectively). But for initial conditions that peak at $k \approx 14$, in some cases, they show decays of $E_{3 D}$ and $E_{2 D}$ that are consistent with predictions for bounded flows (Fig. 4 (b)),

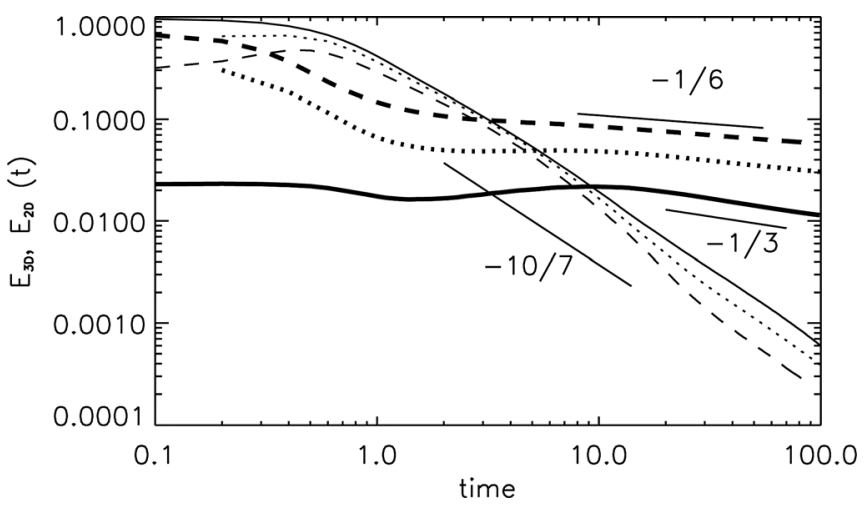

FIG. 9. Energy decay for different initial anisotropies. Thick lines correspond to the energy in $2 D$ modes $\left(E_{2 D}\right)$, while thin lines correspond to energy in $3 D$ modes $\left(E_{3 D}\right)$. Simulations shown are L192HA-1 (solid), L192HA-2 (dotted), and L192HA-3 (dashed), with increasing anisotropy. 
while in others, they show decays as in the unbounded case (Fig. 4(c)). It may be the case that in the presence of helicity, more separation of scales is needed between the initial energy containing scale and the size of the box in order to study unbounded flows (indeed, run L192H-2, which has an initial energy spectrum peaking at $k=30$, shows a decay compatible with $E_{3 D} \sim t^{-10 / 7}$ ). But it is also observed that these decay laws also depend on whether $\mathrm{ABC}$ or random helical initial conditions are used. In the $\mathrm{ABC}$ flow, two-thirds of the initially excited modes are in the $k_{\|}=0$ plane (see Eq. (8)), while random initial conditions excite modes in Fourier space distributed more isotropically, resulting in a smaller percentage of energy in the $k_{\|}=0$ modes when compared with the energy in $k_{\|} \neq 0$. This dependence in the initial ratio of energy in 2D and 3D modes may indicate a stronger coupling between 2D and 3D modes in the presence of helicity (in Sec. $\mathrm{V}$, we will explicitly show how changes in the initial anisotropy affect these results).

On dimensional grounds, the impact of helicity in the coupling can be explained as follows. If decoupling takes place in the limit of fast rotation, it should hold until a time $t^{*} \sim R o^{-2}$, after which higher order terms in the multiple time scale expansion make non-resonant interactions relevant. ${ }^{14,15}$ In non-helical unbounded turbulence, $E \sim t^{-5 / 7}$ and $L \sim t^{1 / 7}$ (under approximate conservation of $I$ ). The Rossby number then decays as
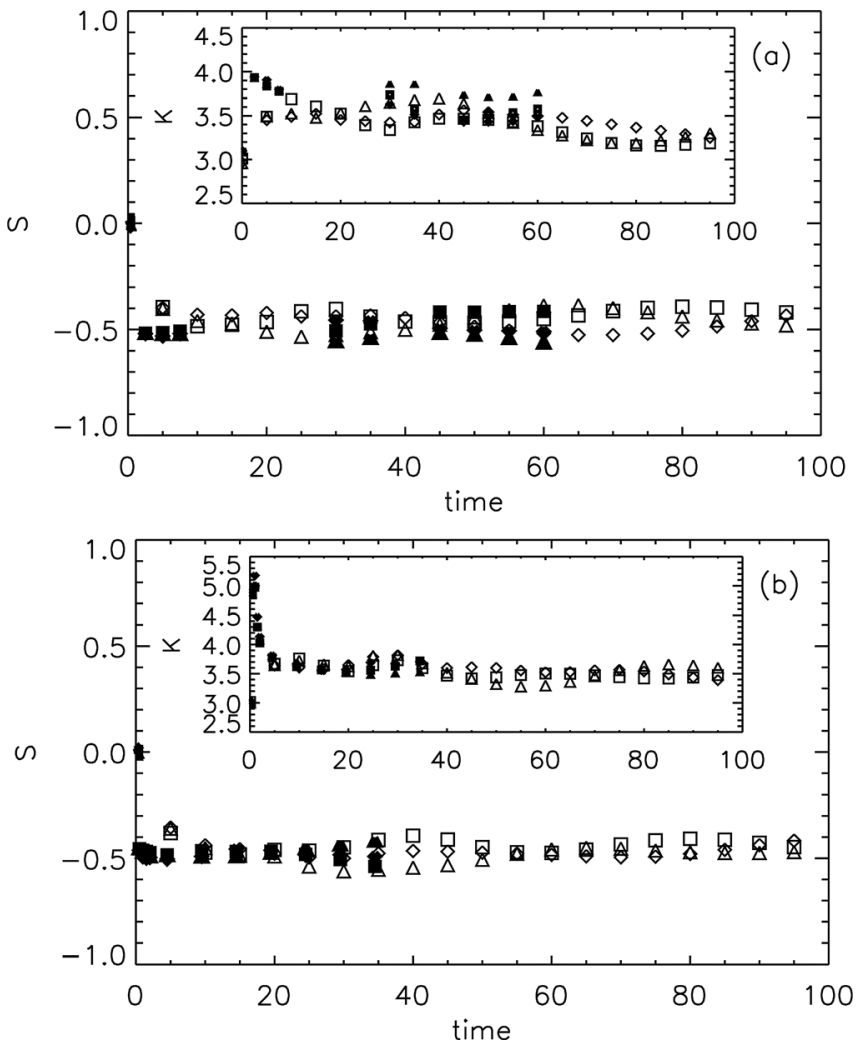

FIG. 10. Evolution of the velocity-derivative skewness for non-rotating runs (a) D512-2 and L96-1 (non-helical) and (b) D512H-2 and L96H-1 (helical). DNS have filled symbols while LES have empty symbols, with squares for $S_{x}$, triangles for $S_{y}$, and diamonds for $S_{z}$. The three components of $S$ oscillate around $\approx-0.5$ independently of helicity content. The insets show the three components of the kurtosis for the same runs using the same labels.

$$
R o=\frac{E^{1 / 2}}{2^{1 / 2} L \Omega} \sim t^{-1 / 2},
$$

and $t^{*}$ grows as $t$. As a result, if decoupling takes place in the freely decaying non-helical case, it can be sustained for long times. The same result $\left(R o \sim t^{-1 / 2}\right)$ is obtained if the argument is refined to consider the $2 \mathrm{D}$ invariants $K$ or $I_{2 D}$ using Eq. (31) or (33), or in the bounded case using Eq. (22). However, in the helical case (e.g., using Eq. (24)), a much slower decay of the Rossby number obtains

$$
R o \sim t^{-1 / 6},
$$

and thus $t^{*}$ grows only as $t^{1 / 3}$.

\section{Spectral evolution and anisotropy}

The isotropic and reduced perpendicular energy spectra are shown in Fig. 6 for LES of rotating flows $(\Omega=10)$ with and without helicity. Energy at large scales grows in all cases, indicating an inverse energy transfer (as also evidenced by a negative flux of energy in that range). Also, the energy spectrum in the helical case (e.g., at the time of maximum enstrophy; not shown) is slightly steeper than in the non-helical case (see, e.g., Refs. 54 and 55).

To further investigate the energy spectral distribution among different directions, we show in Figs. 7 and 8 plots of the axisymmetric energy spectrum $e\left(k_{\|}, k_{\perp}\right)$ for runs L96-6 and $\mathrm{L} 96 \mathrm{H}-3$. Note that to obtain contour levels corresponding
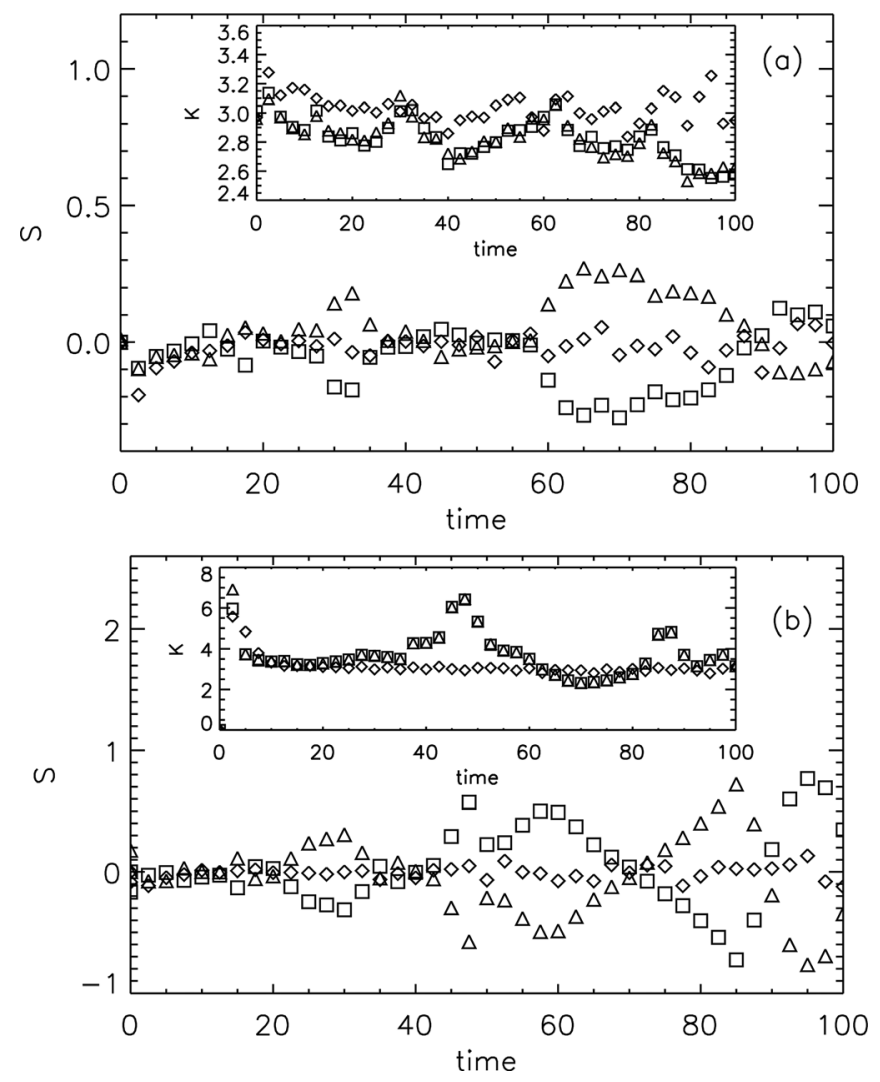

FIG. 11. Evolution of the velocity-derivative skewness for runs (a) L96-6 and (b) L96H-3. Symbols are squares for $S_{x}$, triangles for $S_{y}$, and diamonds for $S_{z}$. The inset shows the evolution of velocity-derivative kurtosis for the same runs. 
to circles in the isotropic case, here and in the following, the axisymmetric energy spectrum is divided by $\sin \theta$, where $\theta=\arctan \left(k_{\|} / k_{\perp}\right)$.

In the case without rotation, the spectrum has an isotropic distribution of energy evidenced by circular contour levels, which maintain their shape as the flow decays (not shown). Alternatively, when rotation is present, the distribution of energy becomes anisotropic with more energy near the $k_{\|}=0$ axis at late times (Fig. 7). This preferential transfer towards slow 2D modes is well known, see e.g., Refs. 3-5. However, for helical rotating flows, there is even more energy near the $k_{\|}=0$ axis (Fig. 8), and energy is also more concentrated at large scales (small $k_{\perp}$ wave numbers), in agreement with our previous observations of a faster increase of integral scales in the presence of helicity.

\section{EFFECT OF INITIAL ANISOTROPY}

As mentioned before, some of the differences observed in the evolution of $E_{2 D}$ and $E_{3 D}$ in helical runs are associated with differences in the initial conditions. In particular, runs with $\mathrm{ABC}$ and with random helical initial conditions differ
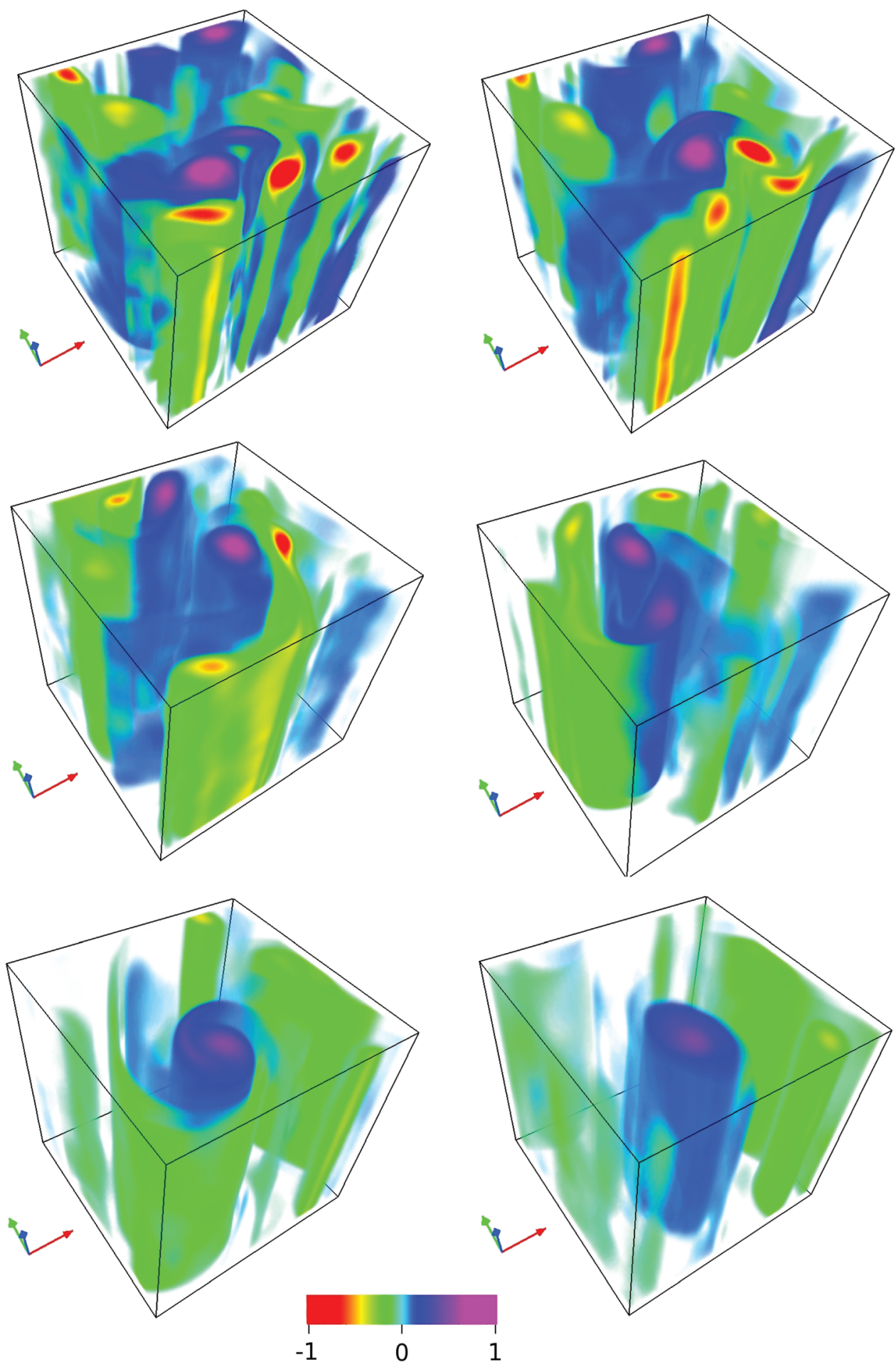

FIG. 12. (Color online) Visualization of $\omega_{z}$ at late times for run $\mathrm{L} 96 \mathrm{H}-3$. From top to bottom and from left to right, the images correspond to $t=42.5,47.5,55$, $70, t=87.5$, and 100 . Note that the four anti-cyclonic vortices at $t=42.5$ merge in pairs and become two larger columnar vortices at $t=47.5$. Eventually, they merge again becoming one column. 
in the fact that the $\mathrm{ABC}$ flow initially has $2 / 3$ of the excited modes in Fourier space in the slow 2D manifold, while in the random case energy is more isotropically distributed.

To further investigate this effect, we consider a set of helical runs with random initial conditions but with increasing initial anisotropy (runs L192HA-1, L192HA-2, and L192HA$3)$. Anisotropy is introduced by weighting the amplitude of all modes with $k_{\|}=0$ with a parameter $\alpha(\alpha=1$ corresponds to the isotropic initial conditions considered before, and $\alpha>1$ corresponds to larger amplitude of the $2 D$ modes relative to the $3 D$ modes). The runs have $\alpha=1$ (L192HA-1), 5 (L192HA-2), and 10 (L192HA-3), resulting in initial ratios of the energy in $2 \mathrm{D}$ to $3 \mathrm{D}$ modes $E_{2 D} / E_{3 D} \approx 0.024,0.626$, and 2.408 , respectively. The runs also have initial energy and helicity spectra peaking at $k=25$, thus allowing us to study unbounded cases with larger scale separation.

Figure 9 shows that $E_{3 D}$ decays approximately as $\approx-10 / 7$ regardless of the anisotropy of the initial conditions, while $E_{2 D}$ changes its decay becoming shallower as anisotropy grows. On the one hand, the isotropic case $(\alpha=1)$ is closer to a $E_{2 D} \sim t^{-1 / 3}$ law, a result consistent with the $2 D$ decay shown in Fig. 4(d). On the other hand, the decay in the most anisotropic case $(\alpha=10)$ is closer to $\sim t^{-1 / 6}$, which is consistent with the decay for helical flows in the case when $K$ is approximately constant; see Eq. (35). Indeed, it was verified that $K$ remains approximately constant during the decay of this run (not shown).

\section{SKEWNESS AND KURTOSIS}

In this section, we consider the time evolution of skewness $S_{i}$ and kurtosis $K_{i}$ of velocity derivatives defined as

$$
\begin{gathered}
S_{i}=\left\langle\left(\frac{\partial u_{i}}{\partial x_{i}}\right)^{3}\right\rangle /\left\langle\left(\frac{\partial u_{i}}{\partial x_{i}}\right)^{2}\right\rangle^{3 / 2}, \\
K_{i}=\left\langle\left(\frac{\partial u_{i}}{\partial x_{i}}\right)^{4}\right\rangle /\left\langle\left(\frac{\partial u_{i}}{\partial x_{i}}\right)^{2}\right\rangle^{2},
\end{gathered}
$$

where $i$ denotes the Cartesian coordinates $x, y$, or $z$.

Figure 10 shows $S$ and $K$ for non-rotating runs D512-2 and L96-1 (non-helical) and D512H-2 and L96H-1 (helical). Only a few times are shown for the DNS runs, to compare with the LES. Overall, the DNS and LES show good agreement. The three components of the skewness fluctuate around $\approx-0.5$, a value observed in experiments ${ }^{56}$ and simulations. ${ }^{57}$ Also, the kurtosis evolves towards a value near 3.5. Helicity does not affect the values of $S$ nor $K$ in the absence of rotation.

When rotation is present, skewness is substantially reduced, with all components of $S$ fluctuating around $S \approx 0$. This is shown in Fig. 11 for runs L96-6 and L96H-3 (DNS show the same behavior and are not shown for clarity). Such a decrease of skewness with decreasing Rossby number has already been reported in simulations. ${ }^{5}$ Anisotropy is also evident, manifested in a distinct behavior of $S_{x}, S_{y}$, and $S_{z}$. While fluctuations of $S_{z}$ are small, $S_{x}$ and $S_{y}$ show large and sudden departures from zero with $S_{x} \approx-S_{y}$ at all times. This anti-correlation between the $x$ and $y$ components can be qual- itatively understood from the quasi-two-dimensionalization of the flow. Indeed, for a purely 2D flow, the incompressibility condition becomes

$$
\frac{\partial u_{x}}{\partial x}=-\frac{\partial u_{y}}{\partial y}
$$

which leads to $S_{x} \approx-S_{y}$. Note, however, that rotating flows differ from 2D flows, e.g., in the asymmetry between cyclones and anti-cyclones.

The kurtosis in the runs with rotation has more fluctuations but seems to evolve towards a value near 3. This is clearer for $K_{z}$, while $K_{x}$ and $K_{y}$ also show signs of quasi-twodimensionalization with $K_{x} \approx K_{y}$ at all times.

Visualization of the flow vorticity indicates that maxima and minima of $S_{x}$ and $S_{y}$ correspond to times when two column-like structures in the flow merge. As an example, Fig. 12 shows the evolution of the $z$ component of the vorticity in run L96H-3. When two columns with the same sign of vorticity merge, intense gradients are created in $u_{x}$ or $u_{y}$, giving rise to an increase in $S_{x}$ or $S_{y}$.

\section{CONCLUSIONS}

In this work, we presented a study of the self-similar decay laws that arise in turbulent rotating flows depending on (1) the characteristic scale of the initial conditions (compared with the size of the box), (2) the presence or absence of helicity in the flow, (3) the values of the Rossby and Reynolds numbers, and (4) the amount of anisotropy in the initial conditions. Phenomenological decay laws were obtained for each case considered, and the decay laws were contrasted with the numerical results from DNS and LES using different flows as the initial conditions.

A large number of power laws were identified. It is well known that rotation decreases the energy decay rate, ${ }^{3,7,10,12,13,24,25}$ and our simulations are in agreement with this result. However, our simulations further show that in the presence of rotation, helicity can further decrease this decay. This is different from the non-rotating case, where helicity does not affect the self-similar decay of energy. This result, together with previous studies in the case of forced rotating flows, ${ }^{1,54,55}$ further confirm that helicity plays a more important role in rotating turbulence than what it does in the isotropic and homogeneous cases.

In the presence of rotation, the decay of enstrophy is well described by phenomenological arguments based on isotropic scaling. This can be expected as enstrophy (as well as helicity) is a small-scale quantity, more isotropic than the energy.

The energy in rotating non-helical flows follows either a decay close to a $\sim t^{-1}$ law (when the integral scale of the flow is close to the size of the box) or a decay slightly steeper than $\sim t^{-5 / 7}$ (when the integral scale is smaller than the size of the box, and the large scale energy spectrum is $\sim k^{4}$ ). Better agreement with power-law decay is obtained when the evolution of 2D modes and 3D modes is considered separately. In that case, the energy in $2 \mathrm{D}$ modes decays close to $E_{2 D} \sim t^{-2 / 3}$, and the 3D modes decay as in the non-rotating case, i.e., close to $E_{3 D} \sim t^{-10 / 7}$. 
These power-law decays can be obtained from phenomenological arguments that consider the energy in 2D and 3D modes separately, that assume approximately constant axisymmetric integrals instead of the isotropic Loitsyanski's integral, and that take into account the slow down in the energy transfer associated with rotation. Note we are not claiming that there is decoupling between $2 \mathrm{D}$ and 3D modes in rotating flows, a topic which is beyond the scope of this work. What we show instead is that the energy in 2D and 3D modes in the simulations decay with different rates, both following power laws, and that considering this in the phenomenological description gives better agreement with the numerical results.

The decay of energy in the presence of rotation and helicity shows further variety. When the integral scale of the flow is close to the size of the box, the energy decay is close to $E \sim t^{-1 / 3}$. This decay can be obtained from phenomenological arguments taking into account the role played by the helicity cascade in further slowing down the energy transfer. In simulations with integral scale smaller than the size of the box, the decay is between $E \sim t^{-5 / 7}$ and $\sim t^{-1 / 3}$. As in the non-helical case, clearer power laws arise if the decay of $E_{2 D}$ and $E_{3 D}$ is considered. In that case, $E_{2 D}$ shows decays between $\sim t^{-1 / 2}$ and $\sim t^{-1 / 6}$, and $E_{3 D}$ shows decays close to either $E \sim t^{-2}$ or $E \sim t^{-10 / 7}$.

The results with helicity seem to be more dependent on scale separation (i.e., on the separation between the initial integral scale of the flow, and the size of the box) and on initial anisotropy. It is worth mentioning that the importance of the initial conditions in the decay of rotating turbulence has been recently pointed out also in experiments. ${ }^{58}$

Finally, we presented a study of the time evolution of the skewness and kurtosis of velocity derivatives. Twodimensionalization of rotating flows leads to an anti-correlation of the $x$ and $y$ components of the skewness, which fluctuate around zero. Large departures of these quantities from this value are associated with merging events of columns in the flow.

\section{ACKNOWLEDGMENTS}

The authors thank fruitful discussions with A. Pouquet and help implementing the LES from D. Rosenberg and J. Baerenzung. Computer time was provided by NCAR and CeCAR. T.T. and P.D.M. acknowledge support from UBACYT Grant No. 20020090200692, PICT Grant No. 200702211, and PIP Grant No. 11220090100825.

${ }^{1}$ H. P. Greenspan, The Theory of Rotating Fluids (Cambridge University Press, Cambridge, 1968).

${ }^{2}$ H. P. Greenspan, "On the nonlinear interaction of inertial waves," J. Fluid Mech. 36, 257 (1969).

${ }^{3}$ C. Cambon and L. Jacquin, "Spectral approach to non-isotropic turbulence subjected to rotation," J. Fluid Mech. 202, 295 (1989).

${ }^{4}$ F. Waleffe, "Inertial transfers in the helical decomposition," Phys. Fluids A 5, 677 (1993).

${ }^{5}$ C. Cambon, N. N. Mansour, and F. S. Godeferd, "Energy transfer in rotating turbulence," J. Fluid Mech. 337, 303 (1997).

${ }^{6} \mathrm{Q}$. Chen, S. Chen, G. L. Eyink, and D. Holm, "Resonant interactions in rotating homogeneous three-dimensional turbulence,” J. Fluid Mech. 542, 139 (2005).
${ }^{7}$ L. Jacquin, O. Leuchter, C. Cambon, and J. Mathieu, "Homogeneous turbulence in the presence of rotation," J. Fluid Mech. 220, 1 (1990).

${ }^{8} \mathrm{P}$. Bartello, O. Métais, and M. Lesieur, "Coherent structures in rotating three-dimensional turbulence," J. Fluid Mech. 273, 1 (1994).

${ }^{9}$ W.-C. Müller and M. Thiele, "Scaling and energy transfer in rotating turbulence,” Europhys. Lett. 77, 34003 (2007).

${ }^{10}$ L. J. A. van Bokhoven, C. Cambon, L. Liechtenstein, F. S. Godeferd, and H. J. H. Clercx, "Refined vorticity statistics of decaying rotating threedimensional turbulence," J. Turbul. 9, 6 (2008).

${ }^{11}$ P. D. Mininni and A. Pouquet, "Helicity cascades in rotating turbulence," Phys. Rev. E 79, 026304 (2009).

${ }^{12}$ C. Morize, F. Moisy, and M. Rabaud, "Decaying grid-generated turbulence in a rotating tank," Phys. Fluids 17, 095105 (2005).

${ }^{13} \mathrm{C}$. Morize and F. Moisy, "Energy decay of rotating turbulence with confinement effects," Phys. Fluids 18, 065107 (2006).

${ }^{14}$ A. Babin, A. Mahalov, and B. Nicolaenko, "Global splitting, integrability and regularity of three-dimensional Euler and Navier-Stokes equations for uniformly rotating fluids," Eur. J. Mech. B/Fluids 15, 291 (1996).

${ }^{15} \mathrm{~L}$. Bourouiba, "Model of a truncated fast rotating flow at infinite Reynolds number," Phys. Fluids 20, 075112 (2008).

${ }^{16}$ P. D. Mininni, P. Dmitruk, W. H. Matthaeus, and A. Pouquet, "Large-scale behavior and statistical equilibria in rotating flows," Phys. Rev. E 83, 016309 (2011).

${ }^{17}$ C. Cambon, R. Rubinstein, and F. S. Godeferd, "Advances in wave turbulence: Rapidly rotating flows,” New J. Phys. 6, 73 (2004).

${ }^{18} \mathrm{~V}$. Borue and S. A. Orszag, "Self-similar decay of three-dimensional homogeneous turbulence with hyperviscosity," Phys. Rev. E 51, R856 (1995).

${ }^{19}$ T. Teitelbaum and P. D. Mininni, "Effect of helicity and rotation on the free decay of turbulent flows," Phys. Rev. Lett. 103, 014501 (2009).

${ }^{20} \mathrm{~A}$. N. Kolmogorov, "On degeneration (decay) of isotropic turbulence in an incompressible viscous liquid," Dokl. Akad. Nauk SSSR 31, 538 (1941).

${ }^{21}$ T. Ishida, P. A. Davidson, and Y. Kaneda, "On the decay of isotropic turbulence," J. Fluid Mech. 564, 455 (2006).

${ }^{22}$ P. G. Saffman, "The large-scale structure of homogeneous turbulence," J. Fluid Mech. 27, 581 (1967).

${ }^{23}$ P. G. Saffman, "Note on decay of homogeneous turbulence," Phys. Fluids 10, 1349 (1967).

${ }^{24} \mathrm{X}$. Yang and J. A. Domaradzki, "Large eddy simulations of decaying rotating turbulence," Phys. Fluids 16, 4088 (2004).

${ }^{25}$ K. D. Squires, J. R. Chasnov, N. N. Mansour, and C. Cambon, "The asymptotic state of rotating homogeneous turbulence at high Reynolds numbers," in Proceedings of the 74th Fluid Dynamics Symposium on Application of Direct and Large Eddy Simulation to Transition and Turbulence, Chania, Crete, Greece, 1994, AGARD-CP-551.

${ }^{26} \mathrm{~W}$. Polifke and L. Shtilman, "The dynamics of helical decaying turbulence," Phys. Fluids A 12, 2025 (1989).

${ }^{27}$ Y. Morinishi, K. Nakabayashi, and S. Ren, "Effects of helicity and system rotation on decaying homogeneous turbulence," JSME Int. J., Ser. B 44, 410 (2001).

${ }^{28}$ M. M. Rogers and P. Moin, "Helicity fluctuations in incompressible turbulent flows," Phys. Fluids 30, 2662 (1987).

${ }^{29}$ D. O. Gómez, P. D. Mininni, and P. Dmitruk, "MHD simulations and astrophysical applications,” Adv. Space Res. 35, 899 (2005).

${ }^{30}$ D. O. Gómez, P. D. Mininni, and P. Dmitruk, "Parallel simulations in turbulent MHD," Phys. Scr. T 116, 123 (2005).

${ }^{31}$ J. Jimenez, A. A. Wray, P. G. Saffman, and R. S. Rogallo, "The structure of intense vorticity in isotropic turbulence," J. Fluid Mech. 255, 65 (1993).

${ }^{32}$ J. Baerenzung, H. Politano, and Y. Ponty, "Spectral modeling of turbulent flows and the role of helicity," Phys. Rev. E 77, 046303 (2008).

${ }^{33}$ J. Baerenzung, P. D. Mininni, A. Pouquet, H. Politano, and Y. Ponty, "Spectral modeling of rotating turbulent flows," Phys. Fluids 22, 025104 (2010).

${ }^{34}$ Y. Li, C. Meneveau, S. Y. Chen, and G. L. Eyink, "Subgrid-scale modeling of helicity and energy dissipation in helical turbulence," Phys. Rev. E 74, 026310 (2006).

${ }^{35}$ G. I. Taylor and A. E. Green, "Mechanism of the production of small eddies from large ones," Proc. R. Soc. London, Ser. A 158, 499 (1937).

${ }^{36}$ S. Childress and A. D. Gilbert, Stretch, Twist, Fold: The Fast Dynamo (Springer-Verlag, Berlin, 1995).

${ }^{37}$ A. Pouquet and G. S. Patterson, "Numerical simulation of helical magnetohydrodynamic turbulence," J. Fluid Mech. 85, 305 (1978). 
${ }^{38}$ R. Monchaux, M. Berhanu, M. Bourgoin, M. Moulin, P. Odier, J.-F. Pinton, R. Volk, S. Fauve, N. Mordant, F. Petrelis, A. Chiffaudel, F. Daviaud, B. Dubrulle, C. Gasquet, L. Marie, and F. Ravelet, "Generation of a magnetic field by dynamo action in a turbulent flow of liquid sodium," Phys. Rev. Lett. 98, 044502 (2007).

${ }^{39}$ L. Skrbek and S. R. Stalp, "On the decay of homogeneous isotropic turbulence," Phys. Fluids 12, 1997 (2000).

${ }^{40}$ L. Biferale, G. Boffetta, A. Celani, A. Lanotte, F. Toschi, and M. Vergassola, "The decay of homogeneous anisotropic turbulence," Phys. Fluids 15, 2105 (2003).

${ }^{41}$ P. A. Davidson, Turbulence: An Introduction for Scientists and Engineers (Oxford University Press, Oxford, 2004).

${ }^{42} \mathrm{O}$. Zeman, "A note on the spectra and decay of rotating homogeneous turbulence," Phys. Fluids 6, 3221 (1994).

${ }^{43}$ Y. Zhou, "A phenomenological treatment of rotating turbulence," Phys. Fluids 7, 2092 (1995).

${ }^{44}$ F. Bellet, F. S. Godeferd, and F. S. Scott, "Wave turbulence in rapidly rotating flows," J. Fluid Mech. 562, 83 (2006).

${ }^{45}$ P. D. Mininni, A. Alexakis, and A. Pouquet, "Scale interactions and scaling laws in rotating flows at moderate Rossby numbers and large Reynolds numbers," Phys. Fluids 21, 015108 (2009).

${ }^{46}$ P. A. Davidson, P. J. Staplehurst, and S. B. Dalziel, "On the evolution of eddies in a rapidly rotating system," J. Fluid Mech. 557, 135 (2006).

${ }^{47}$ T. Teitelbaum and P. D. Mininni, "Large scale effects on the decay of rotating helical and non-helical turbulence," Phys. Scr. T 142, 014003 (2010).
${ }^{48}$ G. K. Batchelor, The Theory of Homogeneous Turbulence (Cambridge University Press, Cambridge, 1953).

${ }^{49}$ P. A. Davidson, "On the decay of Saffman turbulence subject to rotation, stratification or an imposed magnetic field," J. Fluid Mech. 663, 268 (2010).

${ }^{50}$ S. Fox and P. A. Davidson, "Integral invariants of two-dimensional and quasigeostrophic shallow-water turbulence," Phys. Fluids 20, 075111 (2008).

${ }^{51} \mathrm{H}$. Wang and W. K. George, "The integral scale in homogeneous isotropic turbulence," J. Fluid Mech. 459, 429 (2002).

${ }^{52}$ S. B. Pope, Turbulent Flows (Cambridge University Press, Cambridge, 2000).

${ }^{53}$ J. C. André and M. Lesieur, "Influence of helicity on the evolution of isotropic turbulence at high Reynolds number," J. Fluid Mech. 81, 187 (1997).

${ }^{54}$ P. D. Mininni and A. Pouquet, "Rotating helical turbulence. I. Global evolution and spectral behavior," Phys. Fluids 22, 035105 (2010).

${ }^{55}$ P. D. Mininni and A. Pouquet, "Rotating helical turbulence. II. Intermittency, scale invariance, and structures," Phys. Fluids 22, 035106 (2010).

${ }^{56} \mathrm{G}$. K. Batchelor and A. A. Townsend, "The nature of turbulent motion at large wave-numbers," Proc. R. Soc. London, Ser. A 199, 238 (1949).

${ }^{57}$ S. A. Orszag and J. G. S. Patterson, "Numerical simulation of three-dimensional homogeneous isotropic turbulence," Phys. Rev. Lett. 28, 76 (1972).

${ }^{58}$ F. Moisy, C. Morize, M. Rabaud, and J. Sommeria, "Decay laws, anisotropy and cyclone-anticyclone asymmetry in decaying rotating turbulence," J. Fluid Mech. 666, 5 (2011). 